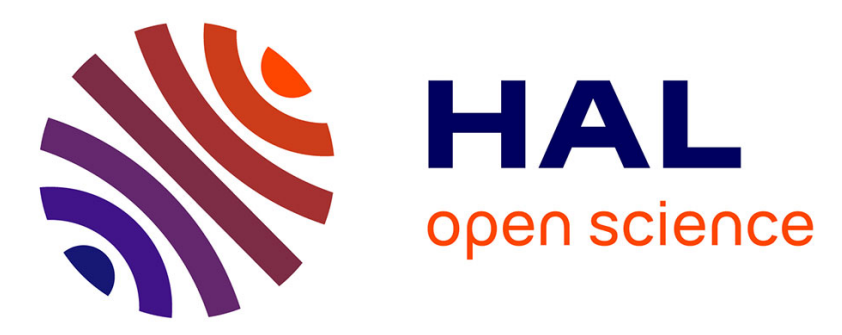

\title{
Numerical modeling of diffusion-controlled phase transformation using the Darken method: Application to the dissolution/precipitation processes in materials
} Sylvie Bordère, S. Glockner

\section{- To cite this version:}

Sylvie Bordère, S. Glockner. Numerical modeling of diffusion-controlled phase transformation using the Darken method: Application to the dissolution/precipitation processes in materials. Computational Materials Science, 2021, 186, 10.1016/j.commatsci.2020.109944 . hal-02942274

\author{
HAL Id: hal-02942274 \\ https://hal.science/hal-02942274
}

Submitted on 4 Dec 2020

HAL is a multi-disciplinary open access archive for the deposit and dissemination of scientific research documents, whether they are published or not. The documents may come from teaching and research institutions in France or abroad, or from public or private research centers.
L'archive ouverte pluridisciplinaire HAL, est destinée au dépôt et à la diffusion de documents scientifiques de niveau recherche, publiés ou non, émanant des établissements d'enseignement et de recherche français ou étrangers, des laboratoires publics ou privés. 


\title{
Numerical modeling of diffusion-controlled phase transformation using the Darken method: application to the dissolution/precipitation processes in materials
}

\author{
S.Bordère ${ }^{\mathrm{a}^{*}}$ and S. Glockner ${ }^{\mathrm{b}}$ \\ ${ }^{a}$ CNRS, University of Bordeaux, Arts et Métiers Institute of Technology, Bordeaux INP, INRAE, I2M \\ Bordeaux, F-33400 Talence, France \\ bBordeaux INP, University of Bordeaux, CNRS, Arts et Métiers Institute of Technology, INRAE, \\ I2M Bordeaux, F-33400 Talence, France \\ * Corresponding author \\ Email address : sylvie.bordere@u-bordeaux.fr (S. Bordère)
}

\begin{abstract}
Many material phase transformations are controlled by mass transport induced by diffusion. To better understand such transformations, numerous modeling strategies at the scale of the moving interfaces exist, with their strengths and weaknesses. Phase field approaches are based on diffuse interfaces that do not require any interface tracking, as opposed to those based on fixed-grid sharp interface tracking. In the case of binary two-phase systems, in this paper we address the key point of the mass balance equation at the interface involving a concentration jump, which determines the interface moving velocity. We propose a unique diffusion equation for both phases and their interface, based on the component's chemical potentials which are continuous throughout the interface and a smooth volume-of-fluid phase representation. This model is achieved in the framework of the Darken method, which involves intrinsic diffusion of components and a drift velocity to which all compounds are subjected. This drift velocity is shown to be that of the interface displacement as well. This methodology is verified for 1D and 3D dissolution/precipitation problems and has a first-order spatial convergence. The 3D simulations of precipitation and dissolution processes of more complex microstructures clearly show a bifurcation of the particle morphology from the initial spherical shape when the diffusion edges of each particle interact with each other. An extension of the diffusion potential to mechanical driving forces should make it possible to deal with mechano-chemical coupling of mass transport.
\end{abstract}


Keywords: diffusion-controlled phase transformation, moving interface, modeling, Darken method, precipitation, dissolution, 3D simulation, Volume-Of-Fluid

\section{Introduction}

The fundamental mechanism of diffusion that controls phase transformations is involved in a wide range of materials processes. Among others, we can mention the solidification of pure liquid processes where energy diffusion is the main driving force, or precipitation/dissolution processes in multi-component systems involving a solid/liquid or a solid/solid interface where mass transport by diffusion has a large impact on microstructure evolutions. This is case in metallurgy during solid state annealing processes, where phase transformations have to be controlled in order to obtain the volume fraction of phases optimizing the mechanical properties [1,2]; and also during transient liquid phase phenomena involving diffusion-induced solidification, leading in some cases to detrimental pore formation $[3,4]$. In this broad field of diffusion processes involving complex coupling of driving forces, we focus in this paper on the particular case of diffusion-controlled phase transformations involving diffusion of components at constant temperature and pressure. In these conditions, during the evolution of the system the local equilibrium at the interface between the two phases is preserved, yielding to a concentration jump at the interface in agreement with thermodynamics. Basically, the underlying physics of such problems is the long-range diffusion of components in each phase with the constraint of the sharp interface equilibrium. The interface displacement is thus related to the conservation of the mass balance at the interface. The approaches based on the Kampmann-Wagner numerical model, which describes nucleation, growth, and coarsening of spherical precipitates, are of great interest in solving such physical phenomena, since they give an insight into microstructure evolution characteristics such as the number of particles and their mean radius [5,6], and for specific times as regards the particle density distribution function [6]. As a complement to these approaches, for which particle morphology remains spherical, there is a need to better understand particle morphology changes during the processes. Several modeling approaches at the interface scale have been developed to deal with this topic. 
The first one is the "phase field" approach, widely developed in materials science [7]. It is based on a diffuse phase function that represents the spatial distribution of the different phases, and on the definition of a free energy functional depending on the phase functions that contains the concentration-dependent parameters [8-11]. The time evolution of the phase function which exhibits the microstructure evolution can thus be obtained from the derivative of the continuous free energy functional with respect to the phase function. As regards the mass transport equation, it can be obtained from the derivative of the free energy functional with respect to component concentrations. While the phase field formalism based on a diffuse interface is not simple, this approach presents the advantage of not tracking a sharp interface, which brings its share of numerical difficulties.

Other approaches consist in solving two types of mass conservation equations, one for the bulk phases and the other for the interface, all equations being coupled. The main difficulty is to model with accuracy the mass transfer and the displacement of the interface and to conserve the total component concentration. Some methods are based on a moving grid and remeshing techniques to follow the interface in order to verify, during the whole simulation, that a point coincides with the interface on which the jump condition is solved. The Landau transformation is used to reach this goal, these methods being adapted to full explicit or implicit approaches, constant to variable diffusion coefficients [12-14] and applied to binary alloys in specific planar, cylindrical or spherical geometries. Remeshing techniques are also used to treat arbitrary 3D mass transfer from single-bubble flows with an Arbitrary Lagrangian Eulerian interface tracking algorithm [15]. Conversely, mass transport equations can also be solved on fixed-grid spatial discretization and coupled with sharp interface tracking, which is widely used for computational fluid dynamic problems. Many methodologies are used for the simulation of these problems such as the front tracking [16], level-set method [17] and Volume-Of-Fluid Piecewise Linear Interface Construction (VOF-PLIC) [18].

Furthermore, some authors have developed specific models based on a single diffusion equation to avoid the explicit treatment of the jump condition for mass transfer through fluid deformable particle interfaces. We can mention the work described in [19,20] where a normalized or scaled concentration helps to remove the jump discontinuity, and the Continuous Species Transfer (CST) model based on volume-averaging proposed in [21-23]. Based on a method originally adapted to thermal solid-liquid phase change problems, a unique diffusion model for the different phases and moving interfaces involved in transient liquid-phase bonding 
is proposed in [24], where a source term is used to take into account the jump condition at the interface.

In this paper, we also aim to develop a unique diffusion model, based on the Darken method [25] (readable in [26]) commonly used in materials science, especially to explain the Kirkendall effect [27], i.e. the lattice shift in solids, if intrinsic diffusivities of atoms are very different. This diffusion model, also called the bi-velocity method, was found to be consistent with the linear irreversible thermodynamics for a monophasic system [28]. It was shown to be efficient for multicomponent systems where the intrinsic fluxes of each component are coupled through the drift velocity $[6,29,30]$. This diffusion model, written through the derivative of diffusion potential, may include different driving forces such as mechanical stress induced by gravity [31] necessary to model segregation in multicomponent mixtures under gravity [32]. This driving force coupling brings a strong potentiality to the Darken-based methodology. This approach has also been shown to be relevant to solve the mass conservation equation at the interface when transfer through this interface is involved [33] for 1D multiphase problems with moving interface. With a view to performing direct simulations of component transfer at the interface, in this work we propose to extend the Darken-based methodology to the two-phase binary system in the framework of the smooth Volume-Of-Fluid (VOF)-based interface advection method. In this way we aim to obtain a continuous single potential for bulk mass transport and transfer through a diffuse interface, allowing us to deal with diffusion-controlled phase transformations involving concentration jumps without having to explicitly calculate the mass conservation equation at the interface.

In the first section of the paper, we present the modeling methodology for each diffusion component (A, B), which leads to a unique diffusion equation through the overall two-phase system composed of 2 bulk phases separated by a diffuse interface region, thus avoiding the specific equation of mass transfer at the interface. Next, the numerical discretization and schemes of the equations are presented in the second section. The third section is devoted to the verification of this methodology with exact solutions for $1 \mathrm{D}$ and $3 \mathrm{D}$ dissolution and precipitation processes. In the last section we present the $3 \mathrm{D}$ simulations of dissolution/precipitation processes of more complex systems involving several particles. 


\section{Modeling}

The mass transport modeling concerns a binary A-B two-phase system (Fig. 1-a). The surface $\Sigma$ represents the interface between the $\alpha$-phase ( $\Omega^{\alpha}$-domain) and the $\beta$-phase ( $\Omega^{\beta}$-domain). The $\alpha$-phase is a solution constituted by A and B components and the $\beta$-phase is a pure phase only constituted by B-component (Fig. 1b).

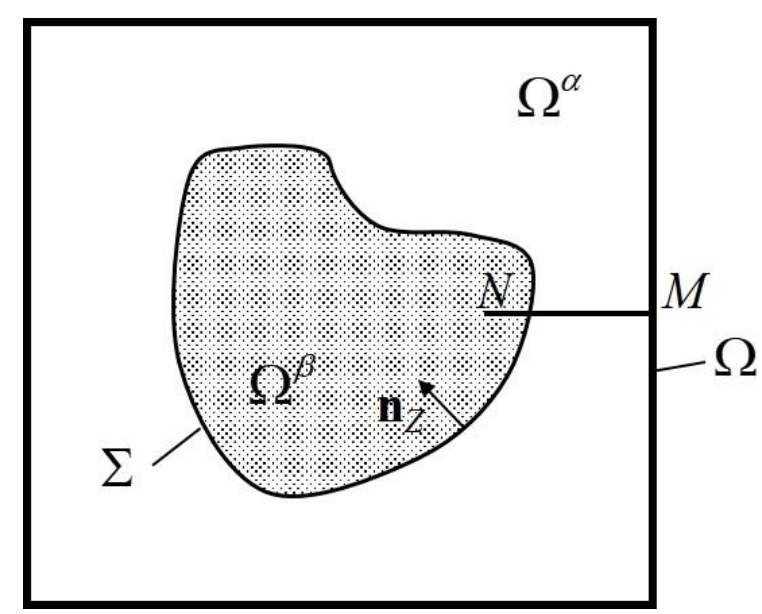

(a)

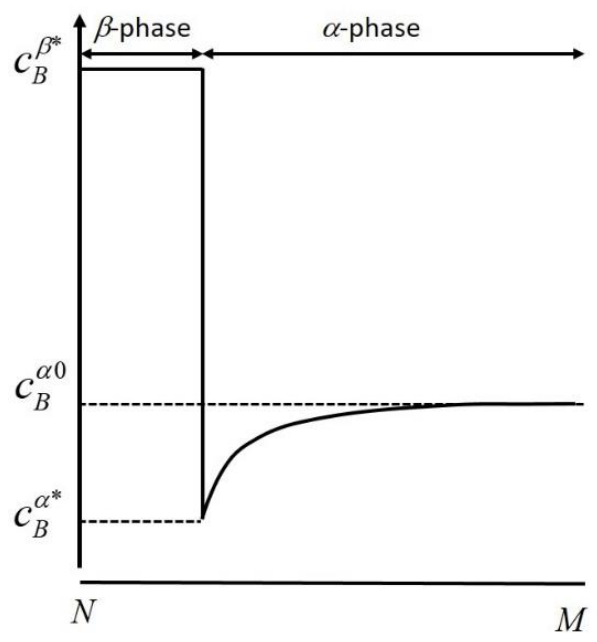

(b)

Fig. 1 : (a) definition of the A-B binary two-phase system, showing along the NM-line: (b) the $\beta$-phase (homogenous), an example of the concentration field in the $\alpha$-phase (A-B solution), and the concentration jump at the interface between the two equilibrium concentrations $c_{k}^{\alpha^{*}}$ and $c_{k}^{\beta^{*}}$ with $k=\mathrm{A}, \mathrm{B} ; c_{k}^{\alpha 0}$ is the initial boundary concentration. Note that the A-B solution is over-saturated in the $\mathrm{B}$-component, corresponding to a $\beta$-phase growth scenario.

The conservation of chemical components $k(k=\mathrm{A}, \mathrm{B})$ specific to the bulk phase domain $\Omega \backslash \Sigma$ is defined by the equation:

$$
\frac{\partial c_{k}}{\partial t}=-\nabla \cdot \mathbf{J}_{k} \text { in } \Omega \backslash \Sigma
$$

where $\mathbf{J}_{k}$ is the total flux of the $k$-component in the volume-fixed frame of reference. If a sharp interface modelling approach is used the $\mathbf{J}_{k}$-fluxes in each phase are solved separately using the inter-diffusion coefficients $D^{\alpha}$ and $D^{\beta}$ corresponding to the $\alpha$-phase and 
$\beta$-phases, respectively, as shown in Fig. 2a. Using the value of the B-component fluxes on both sides of the interface, $\mathbf{J}_{B}^{\alpha / \Sigma}$ and $\mathbf{J}_{B}^{\beta / \Sigma}$, the velocity of the interface $\mathbf{V}_{\Sigma}$ is determined through the mass balance equation $[34,13,18,33]$ :

$$
\mathbf{V}_{\Sigma}=V_{\Sigma} \cdot \mathbf{n}_{\Sigma}=\frac{\mathbf{J}_{B}^{\alpha / \Sigma} \cdot \mathbf{n}_{\Sigma}-\mathbf{J}_{B}^{\beta / \Sigma} \cdot \mathbf{n}_{\Sigma}}{c_{B}^{* \alpha}-c_{B}^{* \beta}} \cdot \mathbf{n}_{\Sigma},
$$

where $\mathbf{n}_{\Sigma}$ is the normal vector to the interface (Fig. 2a) and $c_{B}^{\alpha^{*}}, c_{B}^{\beta^{*}}$ the two equilibrium concentrations.

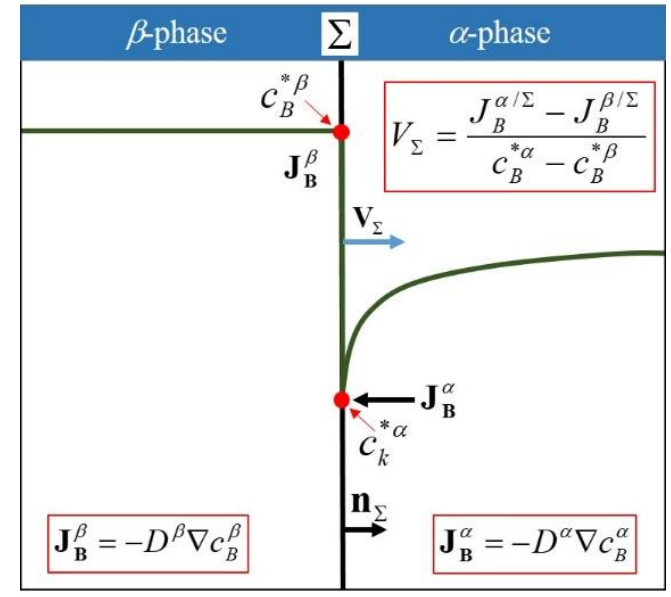

(a)

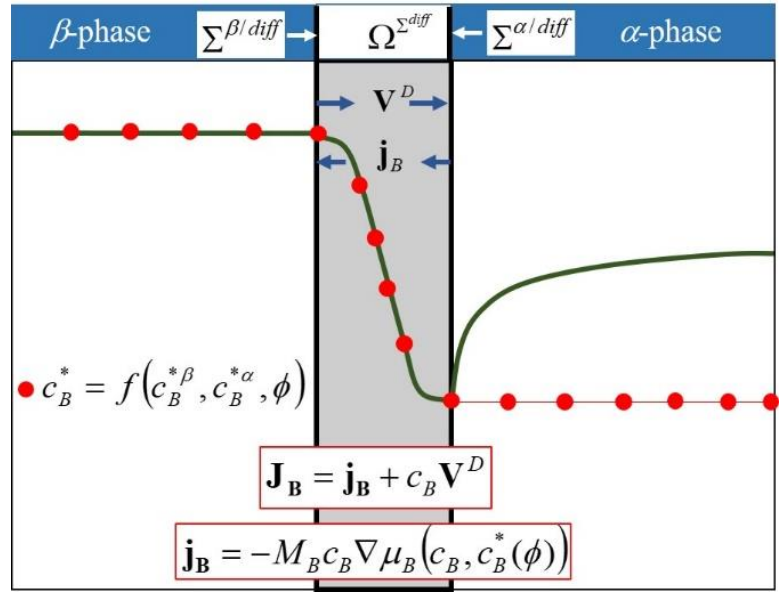

(b)

Fig. 2: Comparison between : a) a sharp interface modelling approach involving the total fluxes $\mathbf{J}_{B}$ and the mass balance equation at the interface to determine the interface velocity $\mathbf{V}_{\Sigma}$; and $b$ ) the proposed methodology based on a unique equation that describes the intrinsic fluxes $\mathbf{j}_{B}$ in the two phases and the diffused interface, and on the Darken velocity that drives the moving interface kinetics.

The aim of the proposed modeling methodology is to overcome the numerical difficulties related to the resolution of the mass balance equation at the sharp interface by considering a diffuse interface approach and by defining a unique transport equation valid in the bulk phases as in a diffuse interface region. This unifying equation results from different modeling steps which are described in the following. In section 2.1, the total fluxes $\mathbf{J}_{k}$ on $\Omega \backslash \Sigma$ are determined thanks to the Darken velocity and an extended chemical potential. In section 2.2 we show that although the chemical potential is continuous at the interface it is not a differentiable function. 
In section 2.3 a smooth phase function is introduced to allow the derivation of the potential and a unique transport equation for the bulk and the interface regions is proposed.

\subsection{Mass transport equation using Darken method}

The proposed approach is based on the theory of multi-component diffusion for simple phases [28-30], which differs from the previous one in that the total flux $\mathbf{J}_{k}$ is decomposed into $i$ ) the intrinsic flux of the $k$-components $\mathbf{j}_{k}$ relative to the lattice-fixed frame of reference and which differ in most cases from one component to another, and ii) the drift flux based on the velocity $\mathbf{V}^{D}$ of the lattice frame relative to the volume frame to which all components are subjected to.

For the binary system this theory corresponds to the Darken one [25,26,34], with $\mathbf{V}^{D}$ being the Darken velocity. The total flux writes:

$$
\mathbf{J}_{k}=\mathbf{j}_{k}+c_{k} \mathbf{V}^{D} \text { in } \Omega \backslash \Sigma
$$

This modeling methodology is thermodynamically consistent [28-30] when combined with volume transport [35] for energy conservation, or when the partial volumes per mole of components are different.

In the following, the defined equations will be restricted to the conditions of constant temperature and pressure and with a same partial volume per mole for the two components in both phases: $\bar{V}_{A}^{\alpha}=\bar{V}_{B}^{\alpha}=\bar{V}_{A}^{\beta}=\bar{V}_{B}^{\beta}=\bar{V}_{m}$.

In these conditions, the total component conservation implies that:

$$
\mathbf{J}_{A}+\mathbf{J}_{B}=0 \text { in } \Omega \backslash \Sigma \text {. }
$$

The Darken velocity can thus be deduced from Eqs. (3) and (4), leading to the relation:

$$
\mathbf{V}^{D}=-\bar{V}_{m}\left(\mathbf{j}_{A}+\mathbf{j}_{B}\right) \text { in } \Omega \backslash \Sigma
$$

The intrinsic fluxes are defined using the extended chemical potential $\mu_{k}[28,30,33,36]$ :

$$
\mathbf{j}_{k}=-M_{k} c_{k} \nabla \mu_{k} \text { in } \Omega \backslash \Sigma,
$$

where $M_{k}$ is the mobility of the $k$-component.

Remark. The extended chemical potential of the $k$-component is defined [28] as: 
$\mu_{k}=\mu_{k}^{c h}+\mu_{k}^{m}+\mu_{k}^{e l}$

where $\mu_{k}^{c h}$ is the pure chemical contribution related to the component concentrations, $\mu_{k}^{m}$ is the mechanical contribution due to pressure, and $\mu_{k}^{e l}$ the electrical contribution resulting from when the charged components are subjected to an electric field.

Through this extended chemical potential, it is possible to couple several driving forces of component diffusion. For instance, in the case where non-negligible surface tensions are involved, the pressure induced by surface tensions giving rise to a pressure jump at the interface can be taken into account through the term $\mu_{k}^{m}=\bar{V}_{m} p$, where $p$ is the local pressure [28]. This pressure jump between the solution and precipitate may lead to non-negligible shifts in the compositions of the individual phases [36]. In our future modeling strategy, the pressure field induced by the surface tensions will be determined from the resolution of momentum conservation equation $[37,38]$.

\subsection{Continuous chemical potential for bulk phases and interface}

In the present work, for the A-B binary two-phase system, at constant temperature $T$ and pressure $p$ and in the absence of any other constraint we have $\mu_{k}^{m}=0$ and $\mu_{k}^{e l}=0$. The chemical potential relative to the $\alpha$-phase can be defined as a function of activity coefficient of the k-component $a_{k}^{\alpha}[1,36]$ by:

$$
\mu_{k}^{\alpha}\left(c_{k}^{\alpha}\right)=\mu_{k}^{0 \alpha}+R T \ln \left(a_{k}^{\alpha}\left(c_{k}^{\alpha}\right)\right) \text { in } \Omega^{\alpha} .
$$

Moreover, at system equilibrium, the concentrations of both components are fixed in the two phases. They are denoted $c_{k}^{\alpha^{*}}$ for the $\alpha$-phase and $c_{k}^{\beta^{*}}$ for the $\beta$-phase, respectively. The interface is thus characterized by concentration discontinuity, whereas the equilibrium chemical potentials $\mu_{k}^{\alpha^{*}}$ and $\mu_{k}^{\beta^{*}}$ of the $k$-component are identical within the two phases. At the two-phase equilibrium we can write in the case of stoichiometric $\beta$-phase:

$$
\mu_{k}^{*}=\mu_{k}^{\beta}\left(c_{k}^{\beta^{*}}\right)=\mu_{k}^{\alpha}\left(c_{k}^{\alpha^{*}}\right)=\mu_{k}^{0 \alpha}+R T \ln \left(a_{k}^{\alpha}\left(c_{k}^{\alpha^{*}}\right)\right) \text { in } \Omega^{\alpha} .
$$

In the case of a diffusion-controlled moving interface process, the interface remains at equilibrium during its evolution, which implies that $c_{k}^{\alpha}\left(\mathbf{x}_{\Sigma}\right)=c_{k}^{\alpha^{*}}$ and $c_{k}^{\beta}\left(\mathbf{x}_{\Sigma}\right)=c_{k}^{\beta^{*}}$ at every 
point $\mathbf{x}_{\Sigma}$ located on the interface (Figs. $1 b$ and 2a). Thus, considering Eq. (9), a continuous chemical potential can be defined, at any position $\mathbf{x}$ of the system:

$\mu_{k}^{\beta / \alpha}(\mathbf{x})=\mu_{k}^{*}+R T \ln \left(a_{k}^{\beta / \alpha}\left(c_{k}^{\beta / \alpha}(\mathbf{x})\right) / a_{k}^{\beta / \alpha}\left(c_{k}^{\beta / \alpha^{*}}(\mathbf{x})\right)\right)$ in $\Omega^{\alpha} \cup \Omega^{\beta} \cup \Sigma$,

verifying that $a_{k}^{\alpha}\left(c_{k}^{\alpha}(\mathbf{x})\right) / a_{k}^{\alpha}\left(c_{k}^{\alpha^{*}}(\mathbf{x})\right)=a_{k}^{\beta}\left(c_{k}^{\beta}(\mathbf{x})\right) / a_{k}^{\beta}\left(c_{k}^{\beta^{*}}(\mathbf{x})\right)=1$ for $\mathbf{x} \in \Sigma$ when the interface is in quasi-equilibrium. This potential is continuous across the interface but is not a differentiable function.

\subsection{Unique transport equation for diffuse interface and ideal solution assumption}

In the context of a smooth VOF method used to model the interface advection [22], we consider the phase function $\phi$ that allows the differentiation of the two phases with $\phi=0$ for the $\alpha$-phase and $\phi=1$ for the $\beta$-phase (Fig. 2b). The part of the domain defined by $0<\phi<1$ corresponds to the diffuse interface volume denoted by $\Omega^{\text {diff }}$ and can be related to an "intermediate phase" characterized by a continuous evolution of the saturation concentration $c_{k}^{*}$ (Fig. 2b) from $c_{k}^{\alpha^{*}}$ to $c_{k}^{\beta^{*}}$ and is defined as follows using the phase function:

$$
c_{k}^{*}=\phi c_{k}^{\beta^{*}}+(1-\phi) c_{k}^{\alpha^{*}}
$$

The local equilibrium of the interface implies that $\mu_{k}\left(c_{k}^{*}(\mathbf{x})\right)=\mu_{k}^{*}$ for $\mathbf{x} \in \Omega^{\text {diff }}$. As a consequence, the potential equation (Eq. (10)) can be defined on the whole system using the phase function. Assuming that the $\alpha$-phase and the diffuse interface verify the condition of ideal solution $a_{k}\left(c_{k}(\mathbf{x})\right)=\bar{V}_{m} c_{k}(\mathbf{x})$ the chemical potential follows:

$$
\mu_{k}(\mathbf{x})=\mu_{k}^{*}+R T \ln \left(c_{k}(\mathbf{x}) / c_{k}^{*}(\phi(\mathbf{x}))\right)
$$

With a phase function sufficiently smooth to express the spatial derivatives at each point of the diffused interface volume $\Omega^{\text {diff }}$, the intrinsic flux equations relative to the two phases in the sharp interface modelling (Eq. (6)) are reduced, when combined with Eq. (12), to a unique equation defined in the overall $\Omega$-domain. This point is illustrated in Fig. 2. As a consequence, the mass conservation equations relative to the two phases (Eq. (1)) can also be reduced to a unique equation in the overall $\Omega$-domain. The transport equation system of component concentrations thus gives rise, with the proposed diffused interface methodology, to: 


$$
\begin{cases}\frac{\partial c_{k}}{\partial t}=-\nabla \cdot \mathbf{j}_{k}-c_{k} \nabla \cdot \mathbf{V}^{D}-\mathbf{V}^{D} \nabla c_{k} \quad k=A, B & \\ \text { with } & \\ \mathbf{j}_{k}=-M_{k} c_{k} \nabla \mu_{k} & \text { in } \Omega^{\alpha} \cup \Omega^{\beta} \cup \Omega^{\text {diff }}, \\ \mu_{k}=\mu_{k}^{*}+R T \ln \left(c_{k} / c_{k}^{*}\right) & \\ \mathbf{V}^{D}=-\bar{V}_{m} \sum_{k=1}^{2} \mathbf{j}_{k} & \end{cases}
$$

where the mobility of the $k$-component is defined through the phase function and the values of the $k$-component mobility corresponding to the $\alpha$-phase and $\beta$-phase, $M_{k}^{\alpha}$ and $M_{k}^{\beta}$ , respectively, as:

$$
M_{k}=\phi M_{k}^{\beta}+(1-\phi) M_{k}^{\alpha}
$$

2.3 Advection of the phase function and computation of mobilities and the equilibrium concentrations

As a final step, after the resolution of Eq. (13), the phase function is advected using the Darken velocity $\mathbf{V}^{D}$ using the equation :

$$
\frac{\partial \phi}{\partial t}=-\mathbf{V}^{D} \nabla \phi
$$

The values of the component mobilities and equilibrium concentrations are then updated with the new phase function $\phi$ using Eq. (11) and Eq. (14).

\section{Numerical resolution}

The proposed model is discretized and solved on a fixed Cartesian staggered grid thanks to the parallel framework of the Notus CFD code [38]. In this context, the scalar variables $\phi, c_{k}$, and $\mu_{k}$, as well as the problem constants $c_{k}^{*}(\phi)$ and $M_{k}(\phi)$ are defined and computed at the center of the mesh cells, whereas the components of the vector variables $\mathbf{j}_{k}, \mathbf{J}_{k}$, and $\mathbf{V}^{D}$ are computed at the center of the cell faces (Fig. 3c). Moreover, the interface does not match the grid (Fig. 3a) 
and is diffused over a few cells by the high gradient of the phase function $\phi$ (Fig. $3 b$ ). The interface is usually plotted thanks to the arbitrary isovalue $\phi=0.5$ (Figs. 3a and 3b).

The equation system Eq. (13-1) is solved as followed: it is split into its diffusion part Eq. (16) and non-conservative form of the advection part Eq. (17), and discretized in time thanks to a first-order forward Euler scheme:

$$
\begin{aligned}
& \left\{\begin{array}{l}
\frac{c_{k}^{l}-c_{k}^{n}}{\Delta t}=-\nabla \cdot \mathbf{j}_{k}^{n} \\
\text { with } \\
\mathbf{j}_{k}^{n}=-M_{k}^{n} c_{k}^{n} \nabla \mu_{k}^{n} \\
\mu_{k}^{n}=\mu_{k}^{*}+R T \ln \left(c_{k}^{n} / c_{k}^{*_{n}}\right)
\end{array}\right. \\
& \left\{\begin{array}{l}
\frac{c_{k}^{m}-c_{k}^{l}}{\Delta t}+c_{k}^{l} \nabla \cdot \mathbf{V}^{D n}=0 \\
\frac{c_{k}^{n+1}-c_{k}^{m}}{\Delta t}+\mathbf{V}^{D n} \nabla c_{k}^{m}=0 \\
\text { with } \\
\mathbf{V}_{D}^{n}=-\bar{V}_{m} \sum_{k=1}^{2} \mathbf{j}_{k}^{n}
\end{array}\right.
\end{aligned}
$$

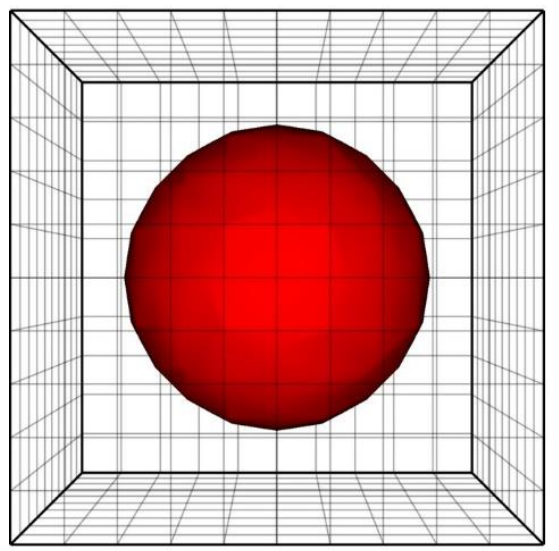

(a)

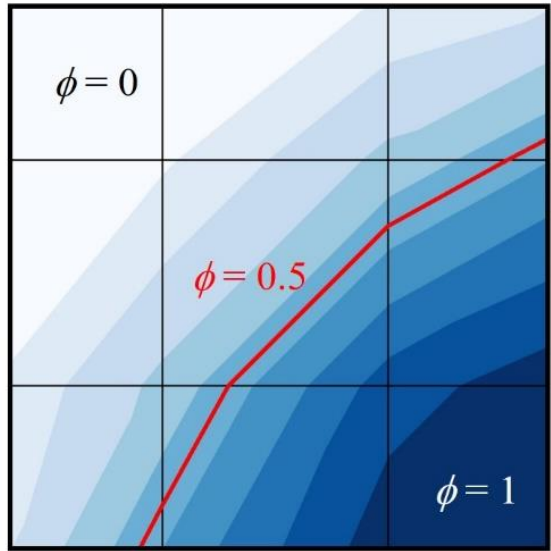

(b)

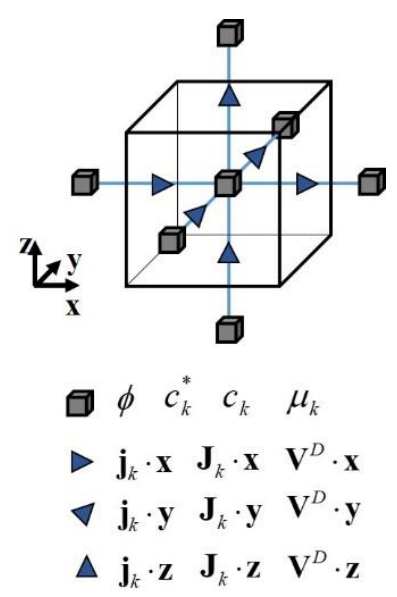

(c)

Fig. 3: (a) Visualization of the phase function $\phi$ described on a uniform grid. This function is able to differentiate the solution $\phi=0$ from the particle $\phi=1$; (b) The location of the interface is arbitrary visualized by the isovalue $\phi=0.5$; (c) Scalar and vector variable locations on a staggered grid. 
A second-order centered scheme is used for the diffusion part with a linear interpolation of the $M_{k} c_{k}$ coefficient to have it on the center of the face cells. A Lax-Wendroff scheme with a Superbee flux-limiter [39] is used to solve the advection part (Eq. (17.2)), as well as the volume fraction phase advection (Eq. (15)). Finally, mobility coefficient and equilibrium concentration are updated respectively thanks to equations (14) and (11).

Remarks. Instead of the Lax-Wendroff TVD scheme other approaches could also be used such as the advection of a level-set function associated with a regularized Heaviside function, or a piecewise linear interface construction method associated to a smoothing function of the reconstructed interface. As it is shown in section 4 the proposed approach is accurate enough and yields results with a very small errors compared to their exact solutions. Moreover, it allows us to efficiently run 3D simulations of several spheres with interface deformations and reconnections. Regarding the initial conditions of the phase function, a subsampling method is used on each cell intersected by the sphere interface. Subsampling has been shown be yield accurate values of the phase function between 0 and 1 on these cells.

\section{Results}

\subsection{Verification of the modeling methodology}

For verification purposes, the proposed methodology is compared with the exact solution of the first equation of system (20) applied to the particle radius growth $r$ developed by Zener [40] for planar $(\lambda=1)$, cylindrical $(\lambda=2)$ and spherical $(\lambda=3)$ geometries, and the following assumptions:

i) homogeneous $\beta$-phase $c_{B}^{\beta}=c_{B}^{\beta^{*}}$ (i.e. no concentration gradient) as, for instance, a pure phase,

ii) Fick's first law for the total flux of diffusion B-component in the $\alpha$-phase $\mathbf{J}_{B}=D \nabla c_{B}$, which states that fluxes are proportional to the concentration gradient, and that every total flux of B-component $\mathbf{J}_{B}$, corresponds to the exact total reverse flux of A component $\mathbf{J}_{A}=-\mathbf{J}_{B}$, satisfying Eq. (3). The inter-diffusion coefficient $D$ is thus written 
using the intrinsic diffusion coefficients $D_{A}$ and $D_{B}$ corresponding to the components A and $\mathrm{B}$, respectively, $[25,26,34]$ as:

$$
D=\bar{V}_{m}\left(c_{A} D_{B}+c_{B} D_{A}\right)
$$

For the ideal solution, the intrinsic diffusion coefficient can be defined by the component mobility through equation $D_{k}=R T M_{k}[25,26,30]$, allowing us to rewrite the inter-diffusion coefficient $D$ as:

$$
\begin{aligned}
& D=R T \bar{V}_{m}\left(c_{A} M_{B}+c_{B} M_{A}\right) . \\
& \text { iii) } \quad \text { constant inter-diffusion coefficient } D, \\
& \text { iv) semi-infinite system insuring zero-flux boundary condition, } c_{B}^{\alpha}(x=\infty, t)=c_{B}^{\alpha 0} .
\end{aligned}
$$

Following these assumptions, the kinetics of the particle radius growth $r$ is defined by:

$$
\left\{\begin{array}{l}
r(t)=\kappa \sqrt{4 D t} \\
\text { where } \kappa \text { is solution of the equation } \\
2 \kappa^{\lambda} I_{\lambda}(\kappa) \exp \left(\kappa^{2}\right)=\frac{c_{B}^{\alpha^{*}}-c_{B}^{\alpha 0}}{c_{B}^{\alpha^{*}}-c_{B}^{\beta^{*}}}=\omega \\
\text { with } \\
I_{\lambda}(x)=\int_{x}^{+\infty} y^{1-\lambda} \exp \left(-y^{2}\right) d y
\end{array}\right.
$$

Note that this solution only depends on the system geometry through the $\lambda$-coefficient and the coefficient of saturation degree, $\omega$, defined with the concentrations $c_{B}^{\alpha^{*}}, c_{B}^{\beta^{*}}$ and $c_{B}^{\alpha 0}$ shown in Fig. 1b. The implicit equation for the $\kappa$-coefficient defined in the second equation of system (20) has been determined numerically without any assumptions on the concentration profile. The kinetics, as well as the concentration profile, of the B-component in the solution domain $\Omega^{\alpha}$ are thus exact for all saturation degrees currently considered. The concentration profile is defined through the $x$-position by the relation $[13,40]$ :

$$
c_{B}^{\alpha}(x, t)=c_{B}^{\alpha 0}+\frac{c_{B}^{\alpha^{*}}-c_{B}^{\alpha 0}}{I_{\lambda}(\kappa)} I_{\lambda}\left(\frac{x-s(t)}{\sqrt{4 D t}}\right),
$$


where $s(t)$ is the position of the two-phase interface at time $t$, the radius being defined by $r(t)=s(t)-s\left(t_{0}\right)$. Note that for $\lambda=1, I_{1}(x)$ is reduced to erfc $(x)$. For a 3D geometry the partial integration of $I_{3}(x)($ Eq. (20.3)) leads to:

$$
I_{3}(x)=\frac{\exp \left(-x^{2}\right)}{x}-\sqrt{\pi} \operatorname{erfc}(x)
$$

giving rise, when introduced in Eq. 20.2 with $\lambda=3$, to another implicit relationship $[34,36]$ for the determination of the kinetics constant $\kappa$ defined as follows:

$$
2 \kappa^{2}\left(1-\sqrt{\pi} \kappa \exp \left(\kappa^{2}\right) \operatorname{erfc}(\kappa)\right)=\omega
$$

Equation system (20) and Eq. (21) are also valid for the case of the dissolution process, but only for the planar geometry. For 3D dissolution the time-dependence of the radius of the precipitate does not verify $r(t)-r_{0}=-K \sqrt{D t}[41,42]$. It should be noted that the exact explicit kinetics does not currently exist. The best approximation is obtained for a stationary interface and an infinite medium assumptions [42], giving rise in the case of small dissolution times $[41,43]$ to:

$$
r(t)=r_{0}+\frac{\omega D t}{r_{0}}+\frac{2 \omega}{2 \sqrt{\pi}} \sqrt{D t}
$$

or in the case of all dissolution times [43] to:

$$
r(t)=\sqrt{r_{0}^{2}+2 \omega D t\left(1+\frac{2 r_{0}}{\sqrt{\pi D t}}\right)} .
$$

The chosen conditions for simulation are congruent with that of the exact solution of Zener, since:

i) the $\beta$-phase is a pure phase constituted by $B$-component $\bar{V}_{m} c_{B}^{\beta}=1$ in $\Omega^{\beta}$.

ii) the intrinsic diffusion of B-component $\mathbf{j}_{B}$ (Eq. (13)), assuming that the $\alpha$-phase is ideal, leads to $\mathbf{j}_{k}=-M_{k} c_{k} \nabla \mu_{k}=-M_{k} R T \nabla c_{k}$ showing that fluxes are proportional to the concentration gradient, thereby consistent with Fick's law. The diffusion of the A-component is currently not considered, i.e. $M_{A}=0$. 
iii) For a two-component system condition ii) gives rise to the inter-diffusion coefficient Eq. (18): $D=R T \bar{V}_{m} c_{A} M_{B}$. To be congruent with the constant inter-diffusion coefficient $D$ for the $\alpha$-phase for a modeling/theory comparison, the mobility of the B-component is considered to be concentration-dependent through the relation:

$$
M_{B}\left(c_{A}\right)=M\left(\frac{1}{\left(\bar{V}_{m} c_{A}+\phi \varepsilon\right)}\right),
$$

where $M$ is a mobility constant and $\varepsilon$ is a low value $(<0.01)$ needed to calculate the mobility when $c_{A} \rightarrow 0$. The simulation kinetics is compared to theory until the concentration at the system boundary $c_{B}(x=L, t)$ remains equal to $c_{B}^{\alpha 0}$.

\subsubsection{D dissolution/precipitation processes}

The simulation of the dissolution process for the planar surface (1D problem) is performed starting from the configuration shown in Fig. $2 \mathrm{a}-\mathrm{T}_{0}$. The thermodynamic and kinetic constants and initial conditions are presented in Table 1.

The Neumann condition is considered for the four boundaries, ensuring zero flux at boundaries and thus mass conservation. The simulations of the dissolution process are performed with a mesh size of $1600 \times 4$ for times up to $t \cdot D / L^{2}=0.01$, and a mesh size of $400 \times 4$-mesh for the long time dissolution process. The time increment is $\Delta t=1 \times 10^{-7} \mathrm{~s}$.

\begin{tabular}{lcccccc}
\hline & $\bar{V}_{m} c_{B}^{\beta^{*}}$ & $\bar{V}_{m} c_{B}^{\alpha^{*}}$ & $\bar{V}_{m} c_{B}^{\alpha 0}$ & $D / L^{2}\left(\mathrm{~s}^{-1}\right)$ & $\omega$ & $\kappa$ \\
\hline 1D dissolution & 1.0 & 0.75 & 0.5 & 1.0 & -1.0 & -0.3578345 \\
\hline 1D precipitation & 1.0 & 0.5 & 0.75 & 1.0 & 0.5 & 0.4327516 \\
\hline 3D dissolution & 1.0 & 0.5 & 0.49 & 1.0 & -0.02 & - \\
\hline 3D precipitation & 1.0 & 0.5 & 0.51 & 1.0 & 0.02 & 0.1099555 \\
\hline
\end{tabular}

Table 1: Thermodynamic and dynamic constants used for the 1D and 3D simulation of the dissolution and precipitation processes. $\bar{V}_{m} c_{B}^{\beta^{*}}$ and $\bar{V}_{m} c_{B}^{\alpha^{*}}$ are the equilibrium molar fractions; initial molar fractions are $\bar{V}_{m} c_{B}^{\beta^{*}}$ for the $\beta$-phase and $\bar{V}_{m} c_{B}^{\alpha 0}$ for the $\alpha$-phase; $\omega$ is the coefficient of saturation degree and $\kappa$ the kinetic coefficient verifying Eq. (20.2) or Eq. (23). 
Fig. 4 presents different characteristic times of the dissolution and precipitation processes showing the displacement of the interface and the corresponding B-component concentration in the $\alpha$-phase. At time $t \cdot D / L^{2}=0.01$, we note that the diffusion edge is far from the right boundary for both processes (Figs. $4 \mathrm{a}-\mathrm{T}_{1}$ and $\mathrm{b}-\mathrm{T}_{2}$ ), thus verifying condition $i v$ ) of the Zener exact solution, $c_{B}^{\alpha}(x / L=1, t)=c_{B}^{\alpha 0}$.

A comparison of the concentration profiles with the exact solutions defined using Eq. (21) is shown in Fig. 5 for three evolution times up to $t \cdot D / L^{2}=0.01$. Note that the computed profiles match the exact ones for both dissolution and precipitation processes, with a high degree of accuracy with respect to the concentration jump at the interface, and the concentration gradient in the $\alpha$-phase. This result shows that the mass transfer dynamic throughout the interface is high enough to obtain local equilibrium, which is driven through the chemical potential defined in Eq. (12). As a consequence, mass transport in the solution is the limited process which imposes the interface kinetics in agreement with the theory framework. 

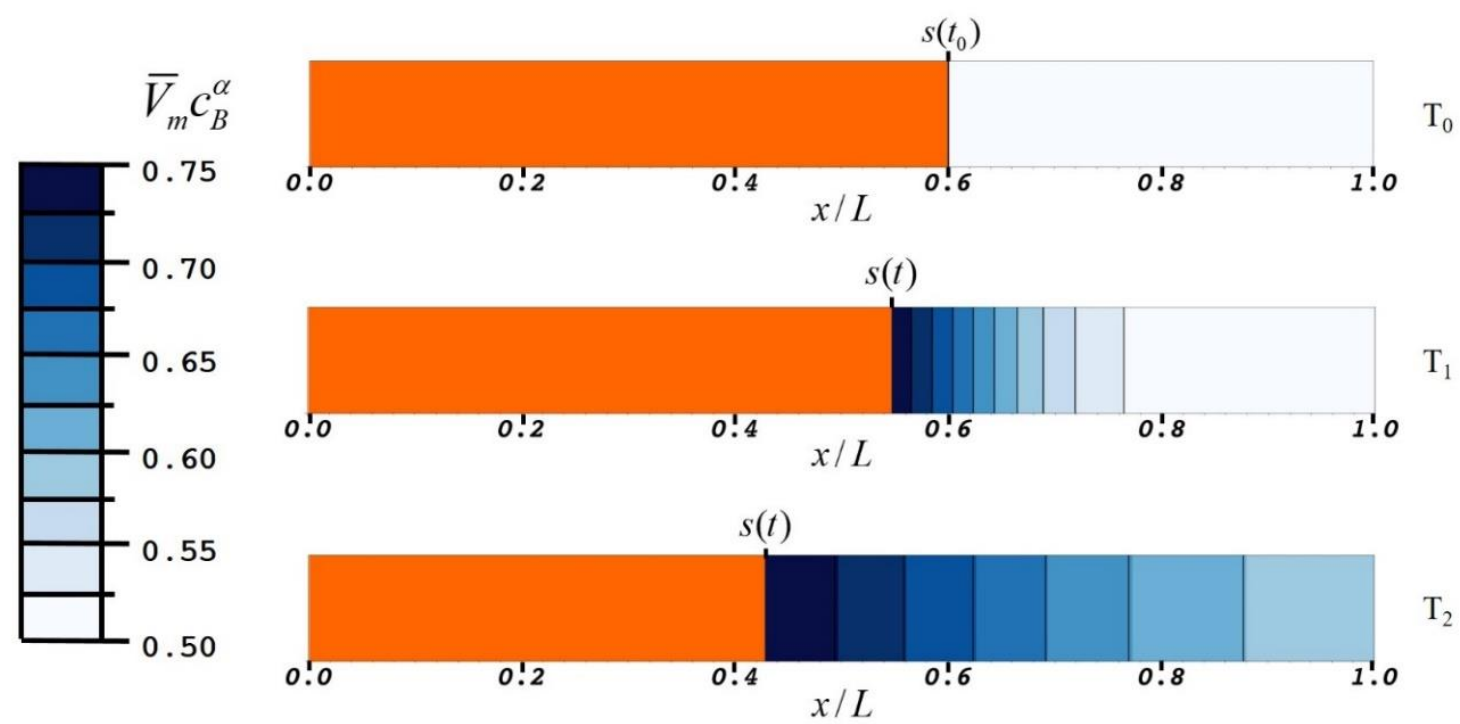

(a)
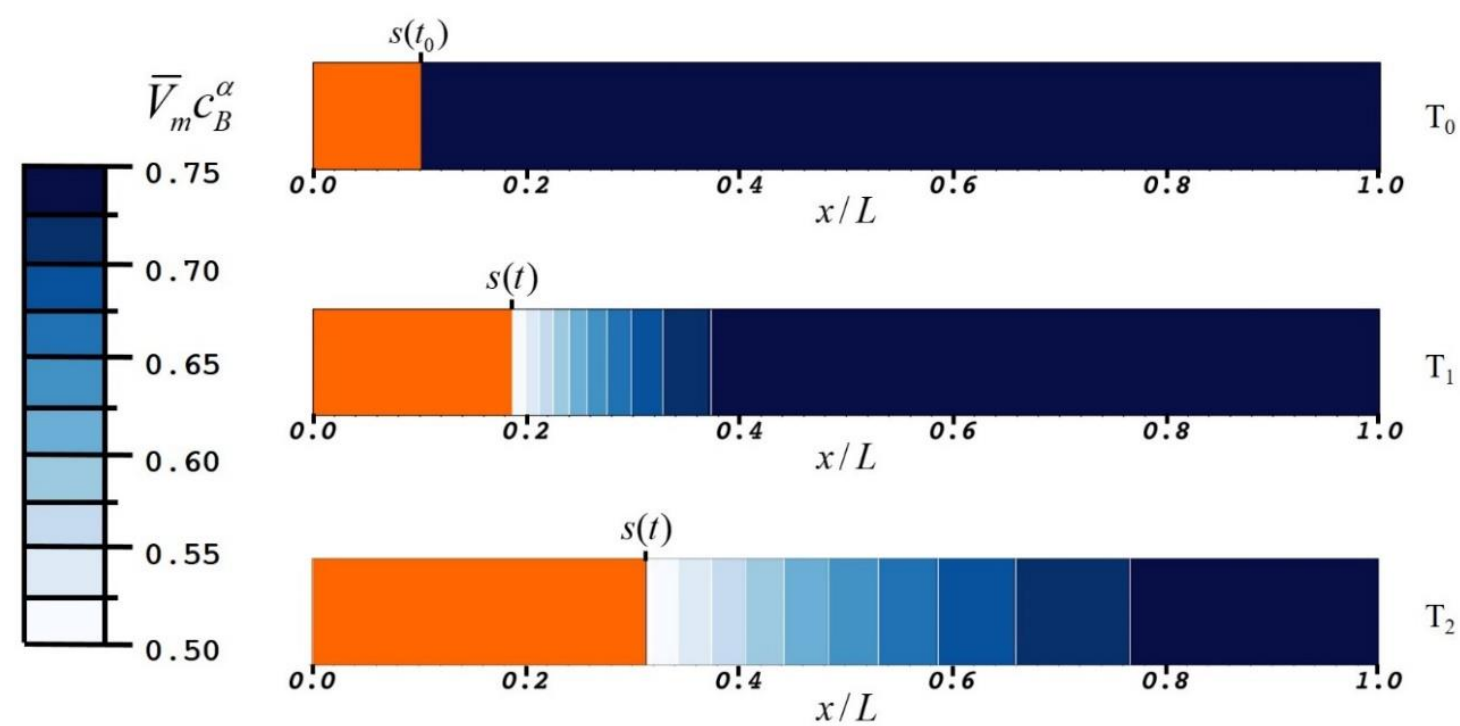

(b)

Fig. 4: 1D simulation of (a) the dissolution and (b) the precipitation processes of a pure solid (in orange) showing the molar fraction field of the $B$-component in the $\alpha$-phase at different evolution times: $\mathrm{T}_{0}, t . D / L^{2}=0 ; \mathrm{T}_{1}, t . D / L^{2}=0.01 ; \mathrm{T}_{2}, t . D / L^{2}=0.06$. 


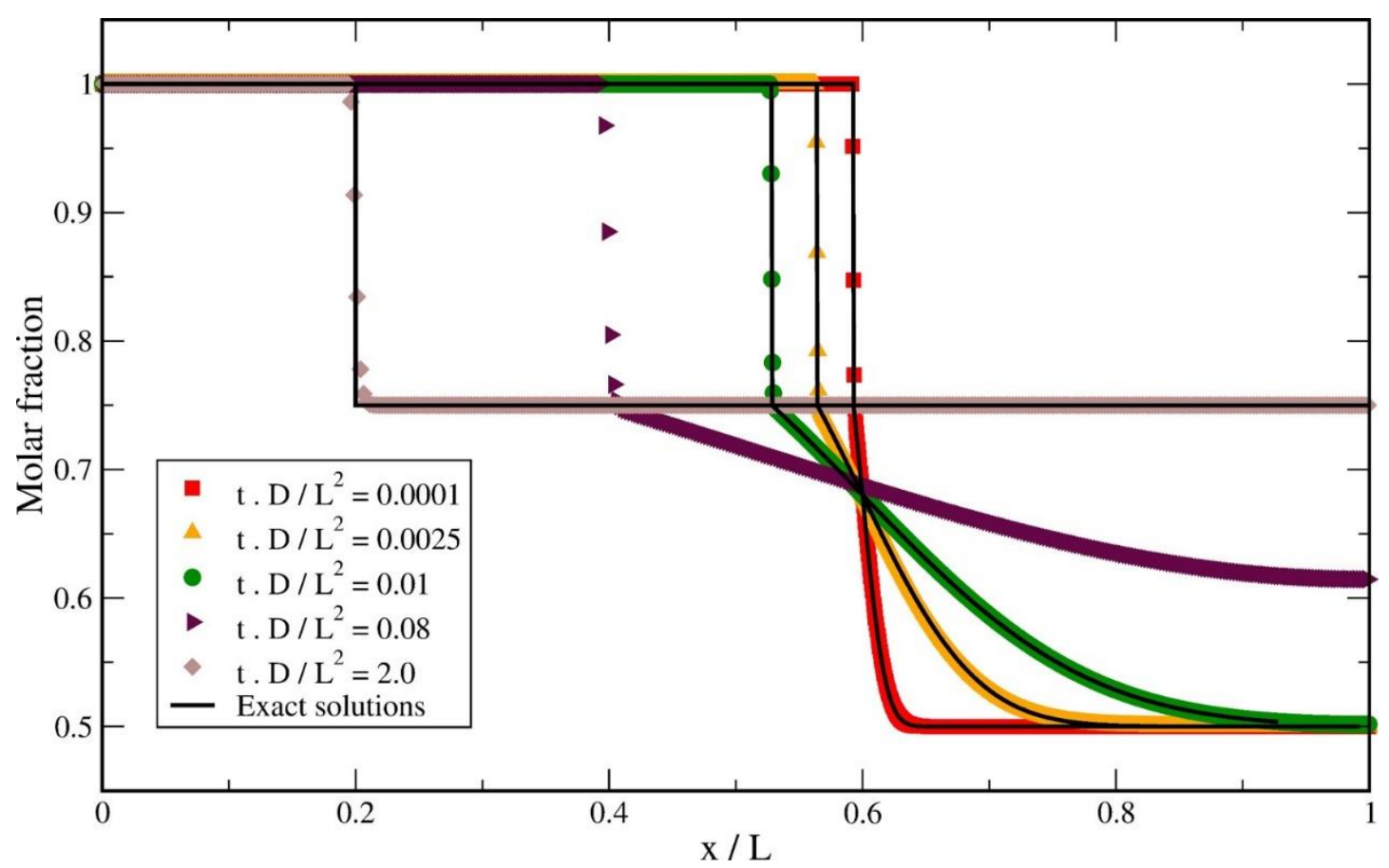

(a)

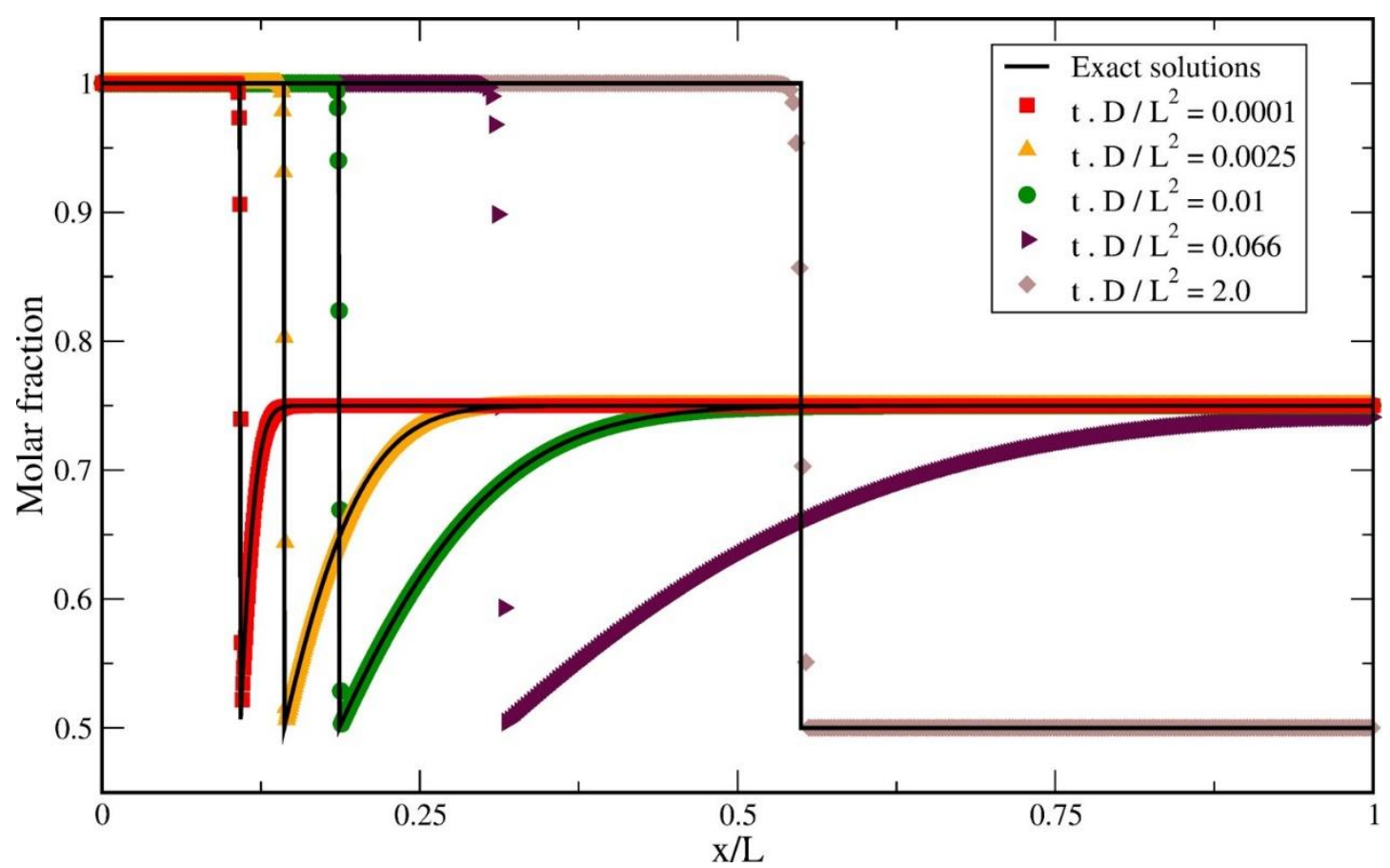

(b)

Fig. 5: Profile evolutions during (a) the dissolution and (b) the precipitation processes until the final stable state. The simulation profiles are compared with the exact Zener profiles (Eq. (21)) for the three dimensionless times $t . D / L^{2}$ up to 0.01 and for the final stable state. 
The kinetics of the interface displacement quantified by the distance $s(t)-s\left(t_{0}\right)=r(t)$ is shown up to the final stable state in Fig. 6 for the dissolution process, and in Fig. 7 for the precipitation process. First, we note that for early times, when the diffusion edge is far from the right boundary, the computed kinetics is obtained with a high accuracy, as can be seen with the magnification of this early time domain (Figs. 6b and 7b). To verify the spatial order convergence of the proposed method, the error on the position of the interface at $t . D / L^{2}=0.01$ is plotted as a function of the mesh size in Fig. 8. A first-order spatial convergence, in accordance with the scheme used, is exhibited for both processes.

When the diffusion edges have reached the boundary, which is the case at time $t . D / L^{2}=0.08$ for the dissolution process (Fig. 5a), and time t.D/ $L^{2}=0.066$ for the precipitation process (Fig. 5b), the theoretical curve established with $c_{B}^{\alpha}(x / L=1, t)=c_{B}^{\alpha 0}$ is no longer valid and thus no comparison with the exact solution is possible. As expected, the end state is obtained when the concentration of B-component is constant throughout the $\alpha$-phase and equal to $\bar{V}_{m} c_{B}^{\alpha^{*}}$, respectively 0.75 and 0.5 for the dissolution and precipitation processes. Note that the exact values of the interface position at equilibrium $s\left(t_{\text {end }}\right) / L=0.2\left(s\left(t_{0}\right) / L=0.6\right)$ for the dissolution process, and $s\left(t_{\text {end }}\right) / L=0.55\left(s\left(t_{0}\right) / L=0.1\right)$ for the precipitation process, are obtained from the equation $s(t) / L=\left(c_{B}^{\text {tot }}-c_{B}^{\alpha^{*}}\right) /\left(c_{B}^{\beta^{*}}-c_{B}^{\alpha^{*}}\right)$ with an accuracy better than $0.1 \%$ for a mesh size of $400 \times 4$ ( $c_{B}^{\text {tot }}$ being the total concentration of B-component into the system). Moreover, the concentration conservation of the B-component in the system during the overall process is around $2 \times 10^{-4} \%$. 


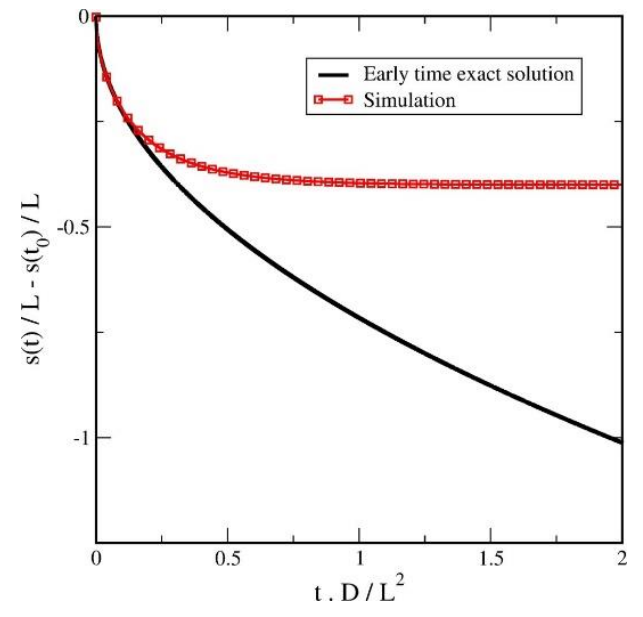

(a)

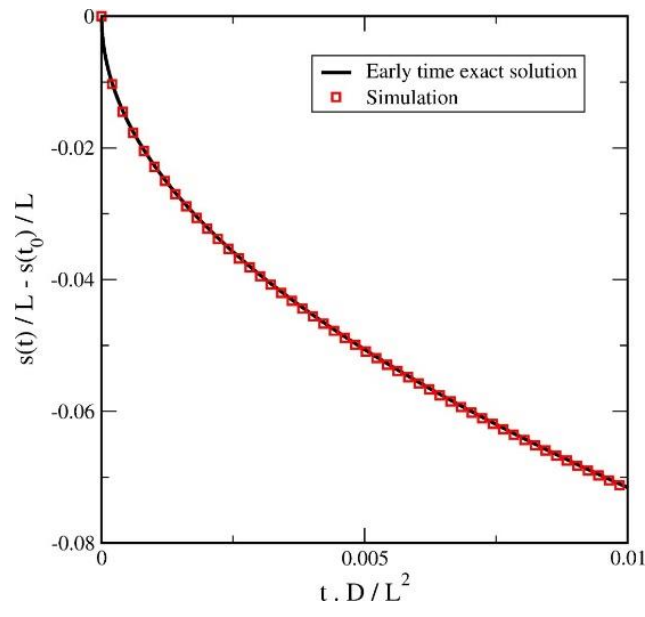

(b)

Fig. 6: Kinetic of interface displacement during the 1D dissolution process for: (a) the overall evolution up to the final stable state; (b) for early time magnification showing a good match between simulation and exact kinetics (Eq. (20.1)).

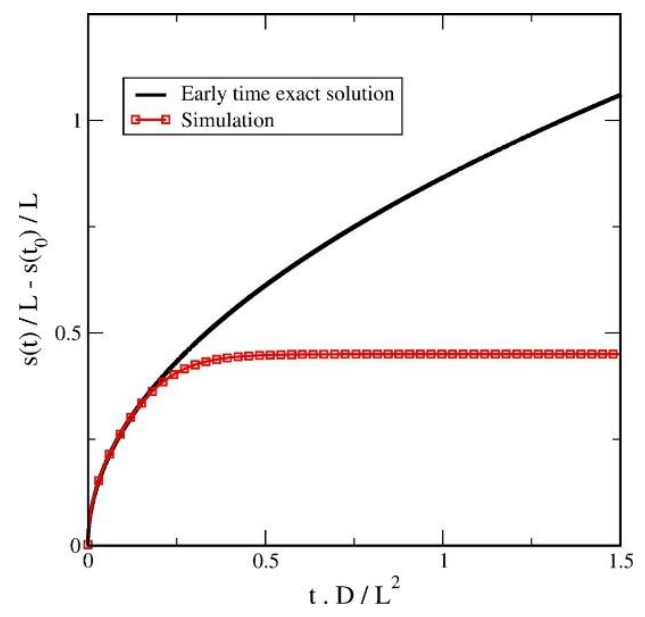

(a)

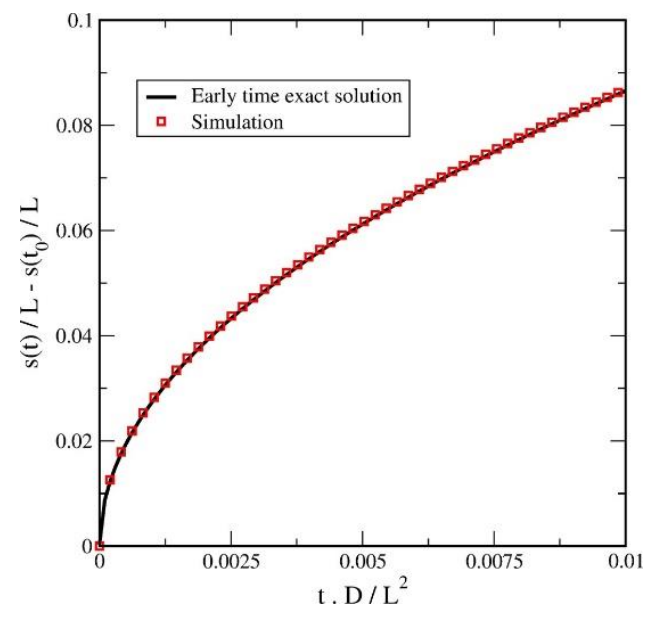

(b)

Fig. 7: Kinetic of interface displacement during the 1D precipitation process for: (a) the overall evolution up to the equilibrium configuration; (b) for early time showing a good match between simulation and exact kinetics (Eq. (20.1)). 


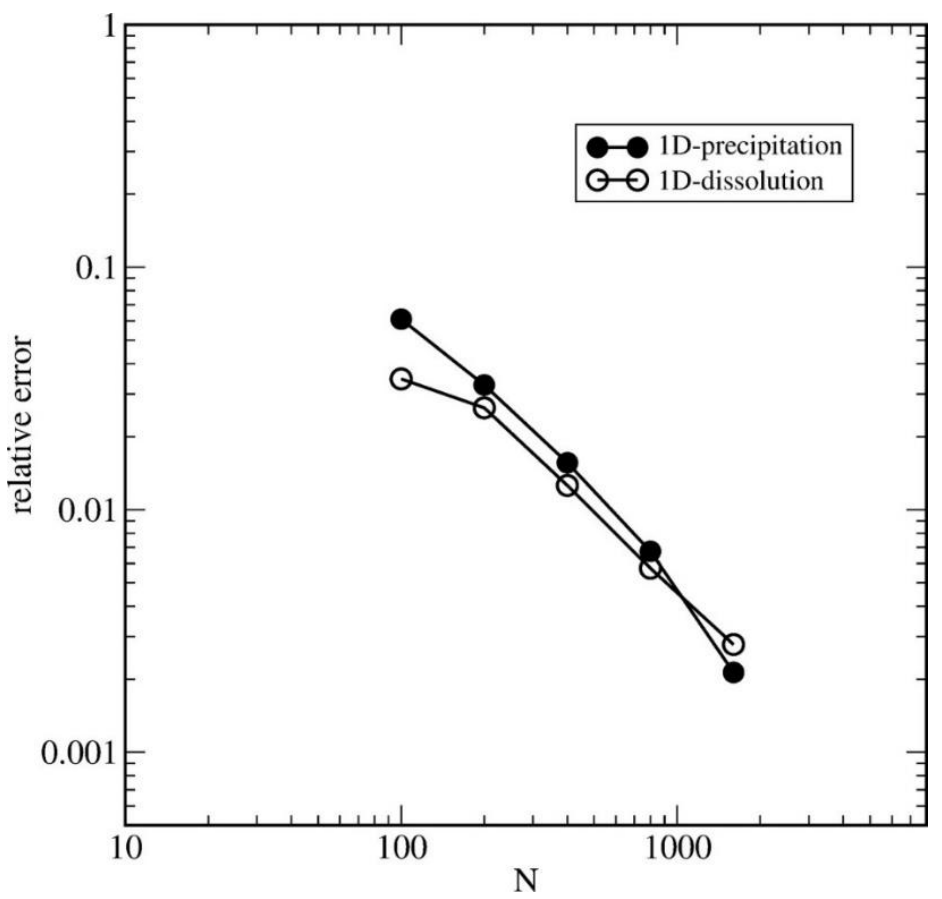

Fig. 8: Relative error $\left(r-r_{t h}\right) / r_{t h}$ of the interface displacement at time $t \cdot D / L^{2}=0.01$ as a function of the number of cells in the mesh $\mathrm{N}$ for both the 1D dissolution and 1D precipitation processes.

To better understand the mass transfer through the interface driven by the gradient of the chemical potential defined in Eq. (12), the scalar variables $c_{B}$ and $\mu_{B}$, as well as the intensity of the vector variables $\mathbf{j}_{k}, \mathbf{J}_{k}$, and $\mathbf{V}^{D}$, are plotted in Fig. 9a. First, we note that except for the chemical potential $\mu_{B}$, all the variables exhibit a jump in the vicinity of the interface. As shown in Fig. $2 \mathrm{~b}$ the Darken velocity intensity $V^{D}$ and the intrinsic flux intensity $j_{k}$ of the B-component in Fig. 9 are nearly constant through the interface. 


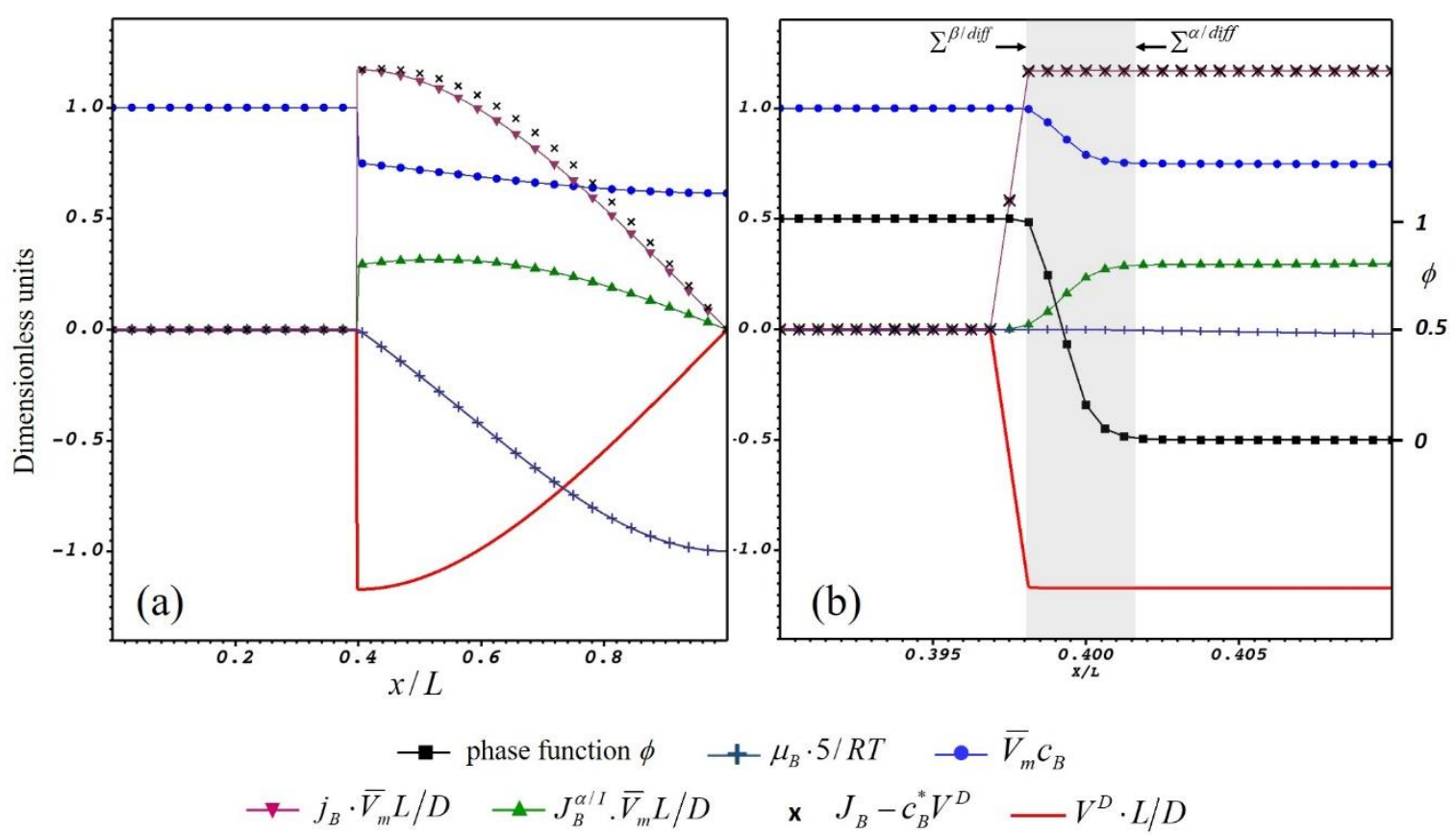

Fig. 9: Characteristics of the scalar variables $c_{B}$ and $\mu_{B}$, and intensities of the vector variables $\mathbf{j}_{k}$, $\mathbf{J}_{k}$, and $\mathbf{V}^{D}$ for the dissolution time $t . D / L^{2}=0.08$ : (a) for the entire system; (b) in the vicinity of the interface identified by the variation of the phase indicator function $\phi$.

Moreover, the intensity $J_{B}-c_{B}^{*} V^{D}$ is constant through the interface. This result for 1D simulation shows that the mass balance through the diffused interface is implicitly considered in this modeling methodology, thereby avoiding the need to solve an additional equation (Eq. (2)). Moreover, the moving velocity of the interface is verified to be the Darken velocity $V^{D}$.

\subsubsection{D precipitation process}

The exact solutions for the spherical particle growth kinetics (system equation (20) with $\lambda=3$ ) is defined with an initial particle radius $r_{0}=0$. Since the simulations have to start from a non-zero particle radius, there are two ways to compare theoretical and numerical simulation results. 
The first one is to considered the concentration field predicted by Eq. (21), with $I_{3}\left(\frac{x-s(t)}{\sqrt{4 D t}}\right)$ calculated from Eq. (22), at the elapse time $t$ for the initial zero-particle radius to reach the radius $r_{0}$ considering Eq. (20.1). The simulation of the spherical particle growth is shown in Fig. 10a and is performed starting from the predicted concentration field at $r_{0} / L=0.025$ using the $\kappa$-coefficient defined from $\omega=0.02$ (see Table 1) and calculated using Eq. (20.2) or Eq. (23). During the evolution, the diffusion edge within the $\alpha$-phase is far from the boundaries, giving rise to spherical iso-values of the of $\mathrm{B}$-component concentration centered on the particle, which are the necessary condition for a comparison with theory. This comparison shows a relative error of $2.2 \%$ and is comparable with the one obtained for the 1D process (Fig. 8).

The second way to compare the simulation and theoretical results is to consider the kinetic equation of the radius squared growth $r^{2}(t)=r_{0}^{2}+4 \kappa^{2} D t$ starting from a constant B-component fraction $\bar{V}_{m} c_{B}^{\alpha 0}$ for the $\alpha$-phase [36]. In this case, the simulation is performed with an initial particle radius $r_{0} / L=0.05$ and a B-component concentration $\bar{V}_{m} c_{B}^{\alpha 0}=0.51$, and the $\kappa$-coefficient is the same as that previously defined for $\omega=0.02$ (see Table 1 ). In this second case, the simulated kinetics of the particle grow until a stable state as shown in Fig. 10b. At the beginning of the process the curve exhibits a straight line allowing us to determine the simulated $\kappa$-coefficient. The obtained value $\kappa_{N U M}=0.1154$ corresponds to a relative error of 4\% compared to the theoretical one (see Table 1) and is in agreement with the spatial convergence study of the 1D process (Fig. 8).

We note that once the diffusion edge reaches the boundaries, the iso-values of the B-component concentration are no longer spherical, leading to a non-isotropic particle growth (concentration map 3 and 3D image 4 in Fig. 10b). The interface displacement is reduced when the distance between the particle and the boundary is short. 


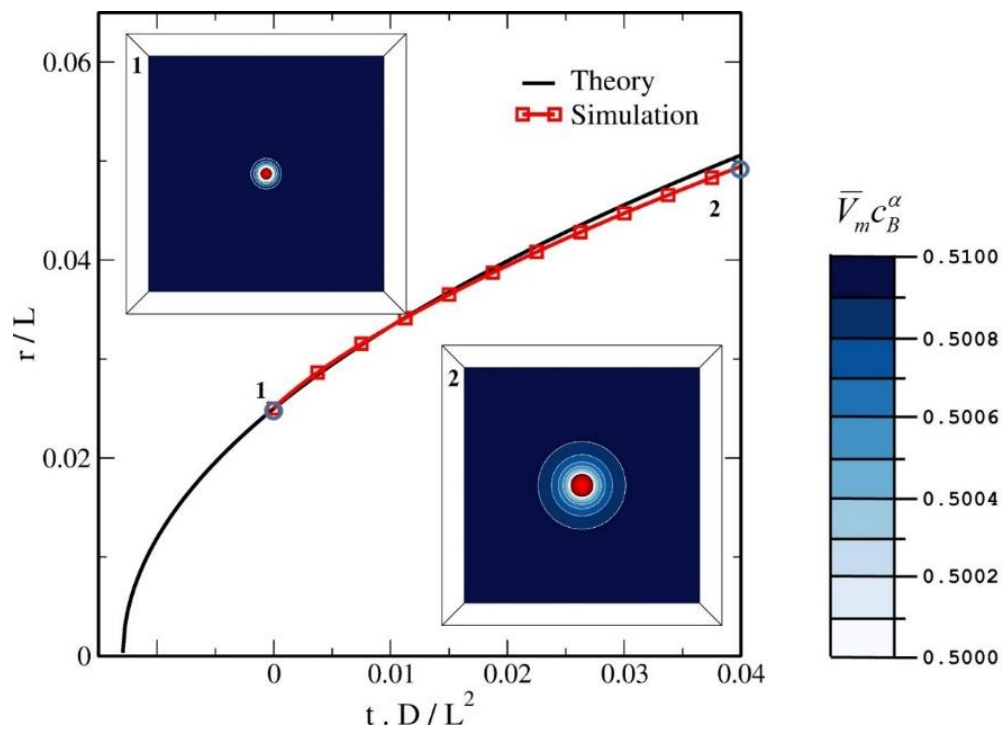

(a)

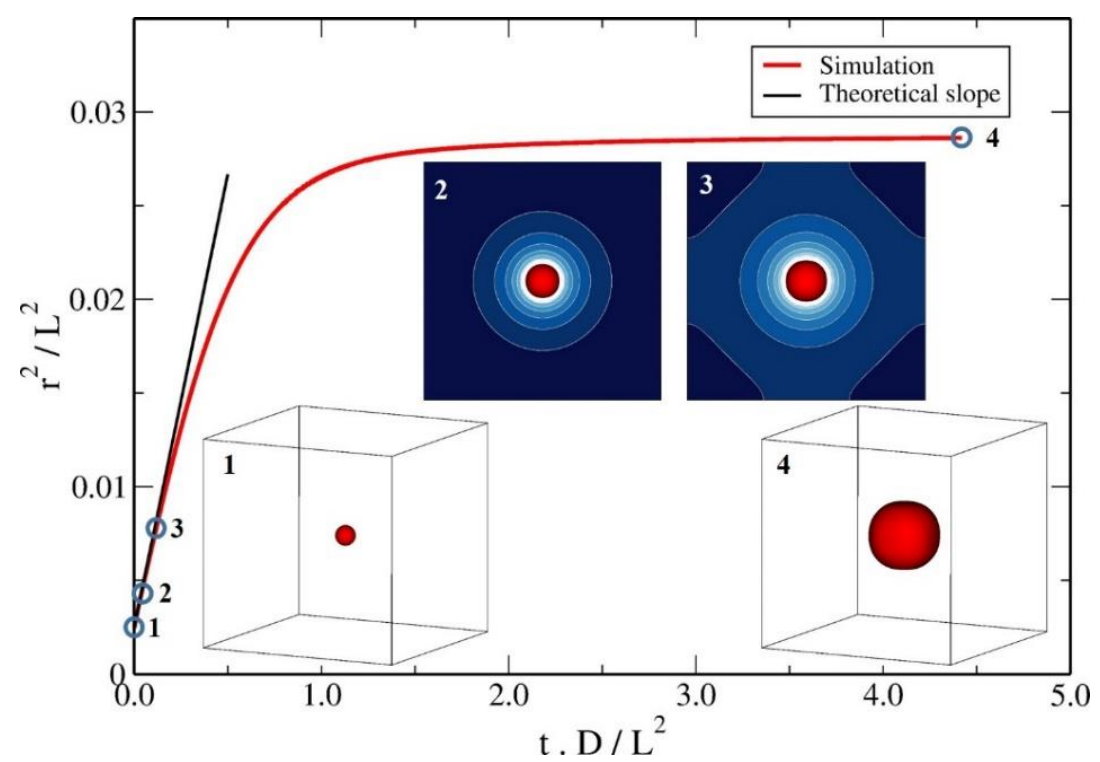

(b)

Fig. 10: Time evolution of the spherical particle during the precipitation process using the saturation degree $\omega=0.02$ (see Table 1) and the characteristic B-component concentration fields. (a) the early radius evolution $r(t) / L$, starting from an initial particle radius $r_{0} / L=0.025$ and its associated concentration field, is compared with the theoretical curve plotted using the $\kappa$-coefficient defined by $\omega$ and $\lambda=3$ (see equations (20.2) with $\lambda=3$ or (23)); a mesh size of $200^{3}$ and a time increment $\Delta t=10^{-7} \mathrm{~s}$ are used; (b) evolution of the particle radius squared $r^{2}(t) / L^{2}$ up to the stable state, starting from an initial particle radius $r\left(t_{0}\right) / L=0.05$ and a B-component concentration $\bar{V}_{m} c_{B}^{\alpha 0}=0.51$; a mesh of size $100^{3}$ and time increment $\Delta t=10^{-6} \mathrm{~s}$ are used. 
To show that the final shape of the particle is not an artifact of the Cartesian discretization, but the result of interactions of the concentration field with boundaries, we have simulated the $\beta$ phase particle growth within a spherical cavity located inside a second domain of $\beta$-phase. The cavity is full of $\alpha$-phase which is oversaturated in B-component (Fig. 11a). In these conditions, the two $\alpha$-phase/ $\beta$-phase interfaces are expected to move in opposite directions while being subtracted from the cubic system geometry. This is precisely what we observe as regards the system at equilibrium shown in Figs. $11 \mathrm{~b}$-c, where the two $\alpha$-phase/ $\beta$-phase interfaces are spherical. It thus highlights that the transformation from spherical shape to smooth cubic shape (Fig. 11d) is induced by the interaction of the concentration field with the cubic system boundaries.

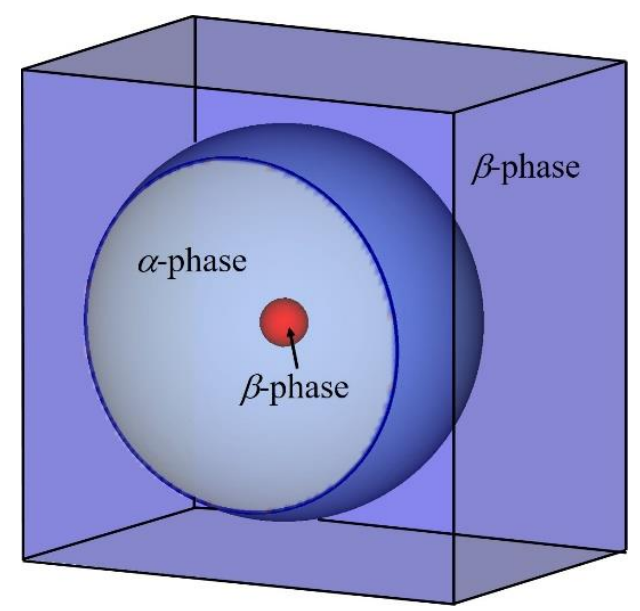

(a)

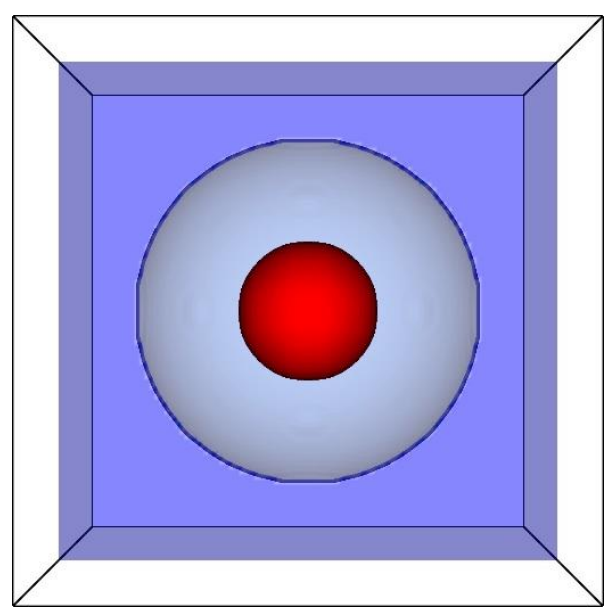

(c)

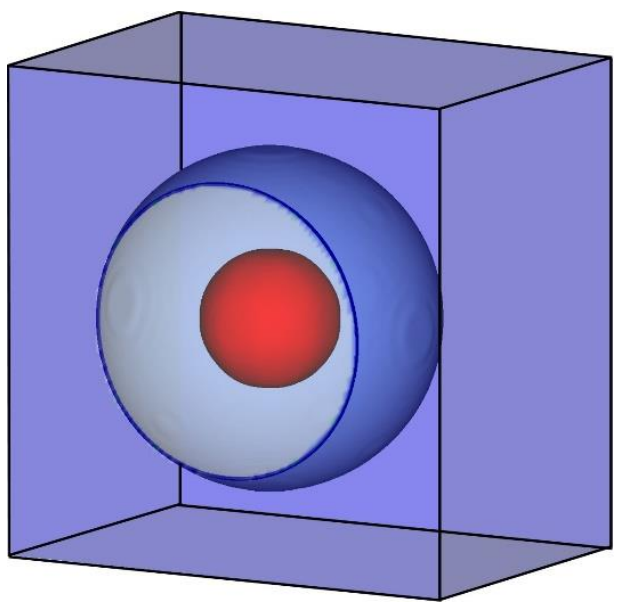

(b)

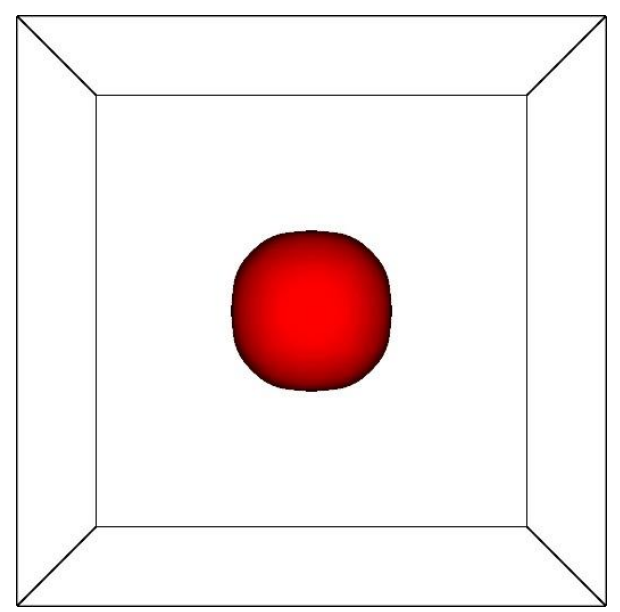

(d)

Fig. 11: Comparison of the $\beta$-phase particle growth within a spherical cavity located inside a second domain of $\beta$-phase and that of the $\beta$-phase growth within the cubic system. The cavity is full of $\alpha$-phase initially oversaturated in B-component $\bar{V}_{m} c_{B}^{\alpha 0}=0.7$. (a) initial configuration; (b) and (c) two views of the final stable state; (d) final stable state of the single particle within the cubic system. 


\subsubsection{D dissolution process}

As detailed at the beginning of section 4.1, which describes the theories of spherical particle precipitation or dissolution, only approximate solutions exist for the 3D dissolution process. Therefore, the simulation of the spherical dissolution starting from a particle radius $r_{0} / L=0.1$ and considering the saturation degree $\omega=-0.02$ (see Table 1 ) is compared to the two approximated solutions defined through Eq. (24) and Eq. (25) and are shown in Fig. (12). We can notice that the simulated and analytical evolutions are coincident at the early times of the dissolution process. However, over time the two analytical kinetics gradually deviate. The approximate solution, which is more accurate for all times of the process (Eq. (25)), results in a kinetic curve that is closer to the simulated one when the concentration field remains spherical around the particle (Point 2 in Fig. 12). The difference between these two curves then slowly increases and is partly the result the interaction of the diffusion edge with the boundary which gives rise to a non-spherical concentration field around the particle (Point 3 in Fig. 12).

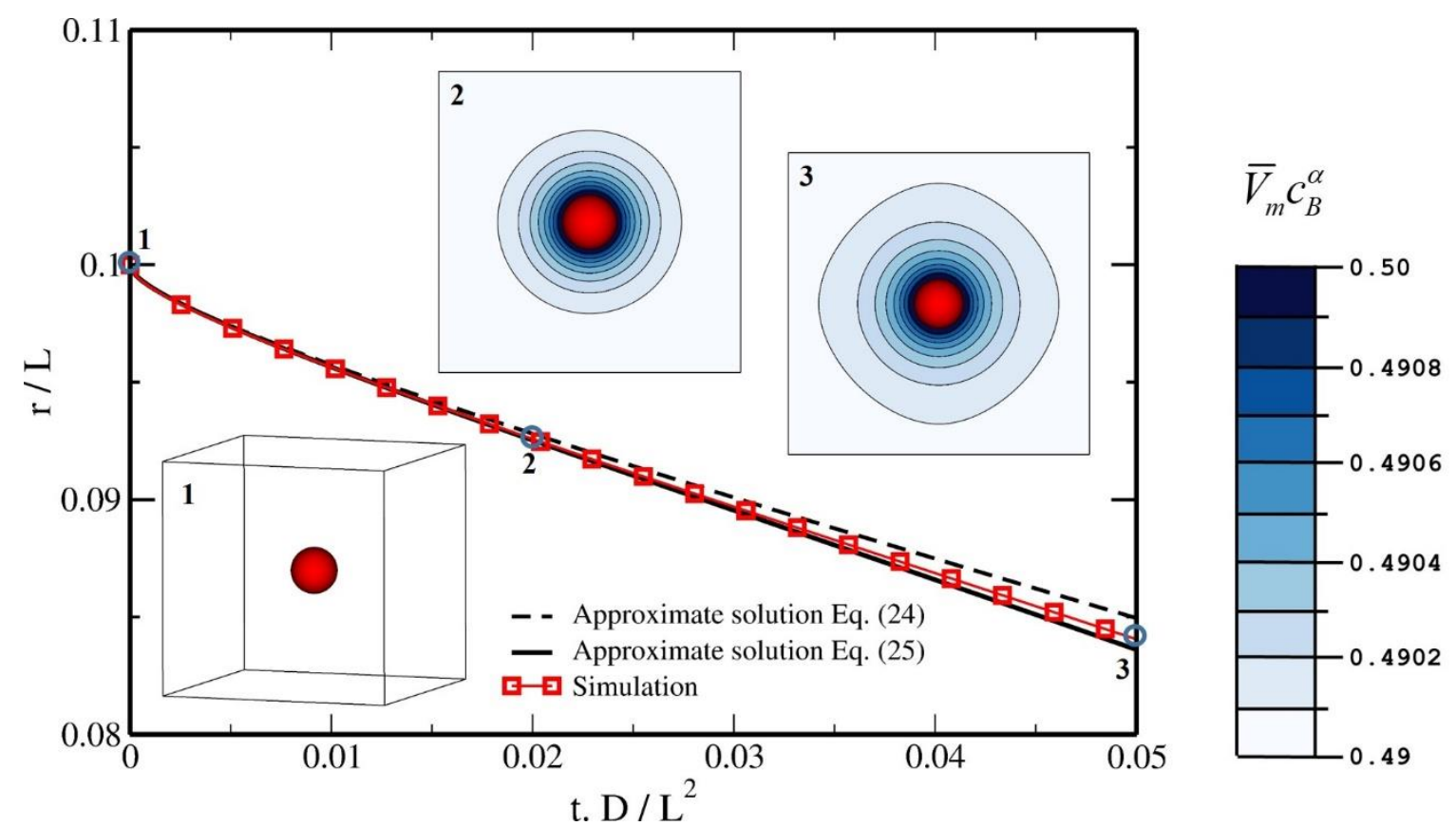

Fig. 12: The early time evolution of the particle dissolution is compared to the approximated solutions defined using the stationary interface assumption; initial particle radius $r_{0} / L=0.1$, saturation degree $\omega=0.02$ corresponding to $\bar{V}_{m} c_{B}^{\alpha 0}=0.49$ (see Table 1); a mesh of size $200^{3}$ and time increment $\Delta t=5 \times 10^{-7} \mathrm{~s}$ are used. 


\section{2- Simulation of complex particle arrangements}

In this section we aim to illustrate the feasibility of the proposed modeling in simulating the dissolution/precipitation processes of complex particle arrangements that are more representative of experimental systems. So, for simulations of dissolution and precipitation processes we considered an arrangement of seven $\beta$-phase particles of spherical shape randomly distributed within the cubic system. The equilibrium molar fractions considered are those defined in Table 1.

In the case of the precipitation process, the particles have the same initial radius $r\left(t_{0}\right) / L=0.05$ (Fig. 13), and the $\alpha$-phase solution is oversaturated in B-component $\bar{V}_{m} c_{B}^{\alpha 0}=0.55$. Fig. 13a presents the kinetic evolution of the volume fraction of the $\beta$-phase up to the stable state. As regards the 3D morphology evolution of the particles (Fig. 13a), as for the single particle process (Fig. 10), the particles remain spherical until the surrounding concentration fields induced by their own precipitation interact with each other. Fig. 13b- $\mathrm{T}_{1}$ shows the first moments where the concentration fields relative to two neighboring particles interact in the plane $z / L=0.35$ defined in Fig. 13b-T $T_{0}$ with the initial particle configuration. From that moment, the particles grow anisotropically until the final state, preferably in directions where other particles are distant (see Figs. 13a, 13b- $\mathrm{T}_{2}$ and $13 \mathrm{~b}-\mathrm{T}_{3}$ ). 


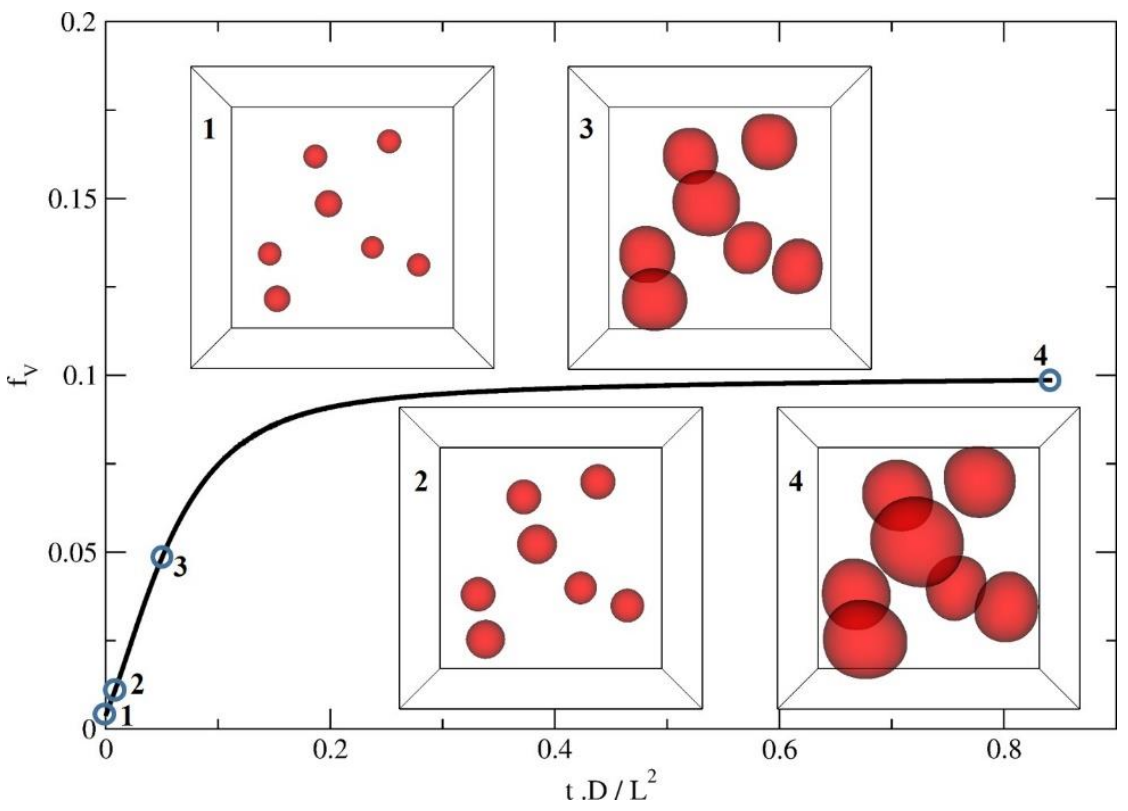

(a)

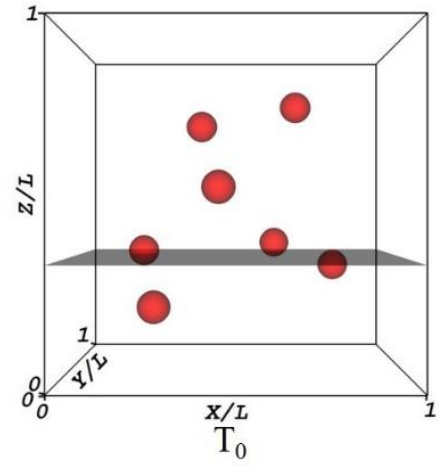

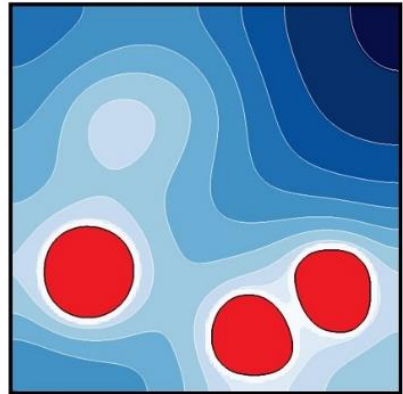

$\mathrm{T}_{2}$

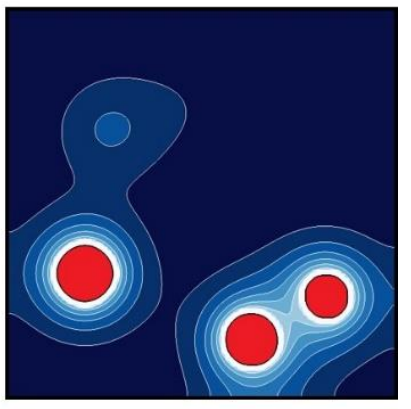

$\mathrm{T}_{1}$

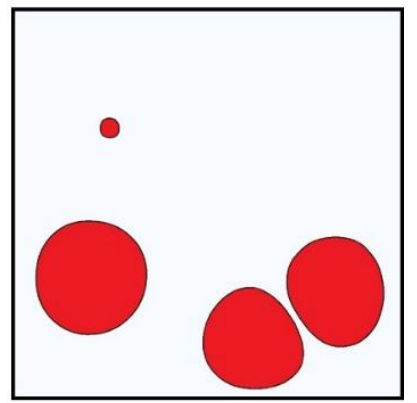

$\mathrm{T}_{3}$

(b)

Fig. 13 (a) Kinetic evolution of the volume fraction of $\beta$-phase and microstructure evolution; (b) characteristics of the concentration fields of B-component relative to the $\alpha$-phase solution during the precipitation process involving 7 particles of spherical shape and identical initial radius $r\left(t_{0}\right) / L=0.05$. The concentration fields of B-component $\bar{V}_{m} c_{B}^{\alpha}$ are drawn in the plane $z / L=0.35$ shown in the initial configuration $\mathrm{T}_{0}$ for different times $t . D / L^{2}: 0.008$ in $\mathrm{T}_{1}, 0.05$ in $\mathrm{T}_{2}$ and 0.8 in $\mathrm{T}_{3}$; simulation was performed using a mesh size of $100^{3}$ and time increment $\Delta t=10^{-6} \mathrm{~s}$. 
In the case of the dissolution process, the particles have the same but larger $r\left(t_{0}\right) / L=0.15$ initial radius (Fig. 14). This particle size means that two of them overlap and intersect the system boundary (bottom right of the 3D configuration in Fig. 14a). The equilibrium concentration $\bar{V}_{m} c_{B}^{\alpha^{*}}=0.5$ is different here from the $1 \mathrm{D}$ dissolution case defined in Table 1, and the $\alpha$-phase solution is undersaturated in B-component $\bar{V}_{m} c_{B}^{\alpha 0}=0.45$. Fig. 14 a represents the kinetic evolution of the volume fraction of the $\beta$-phase up to the stable state and some typical evolving 3D morphologies. As for the precipitation process described before, the concentration fields induced by each particle dissolution rapidly interact (Fig. 14b- $\mathrm{T}_{1}$ ), leading thereafter to anisotropic particle shapes; and the particle interface moves faster in directions where other particles or boundaries are distant (see Fig. 14a and 14b- $\mathrm{T}_{2}$ ). Finally, note that for t.D $/ L^{2}=0.25$ (point 4 in Fig. 14a), the single particles are close to their equilibrium size in comparison with the final state (point 5 in Fig. 14a), whereas the overlapping particles forming like a cylinder continue to dissolve significantly. This result is in agreement with a previous theoretical study that showed a complete dissolution time of cylindrical particles which was greater than that of spherical ones [44]. 


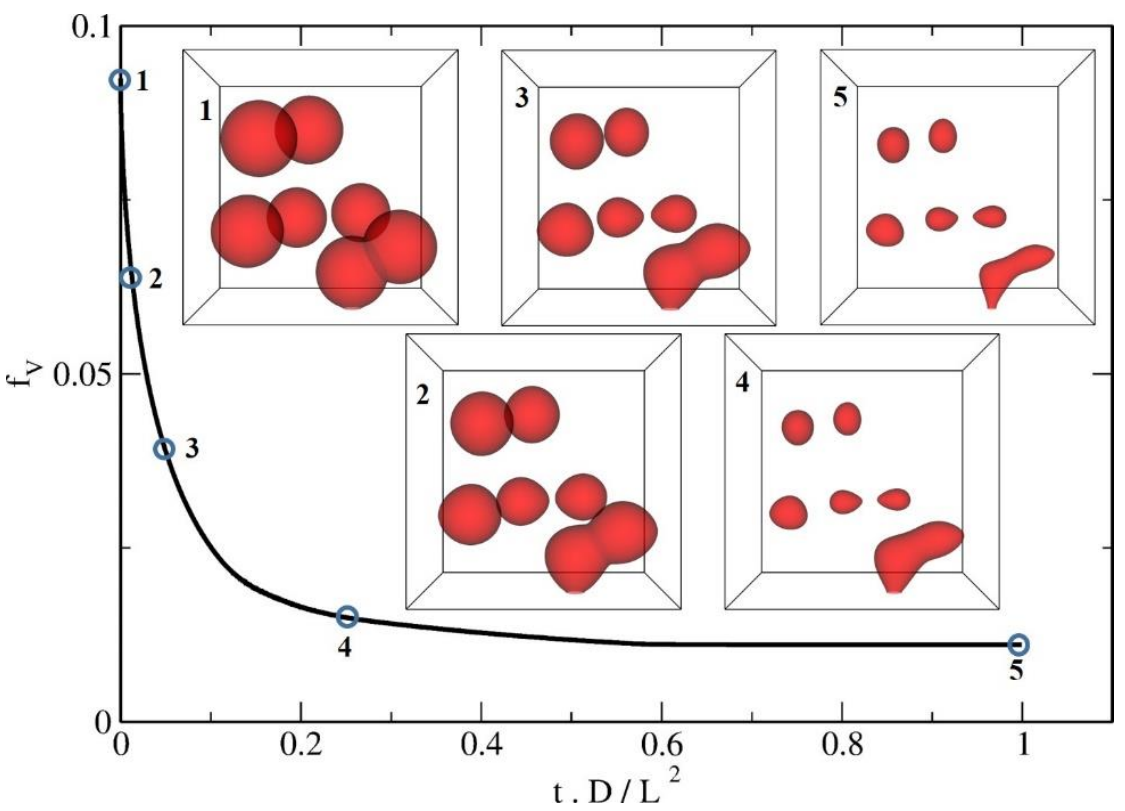

(a)

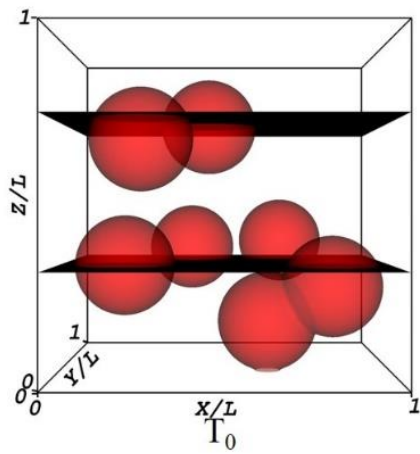

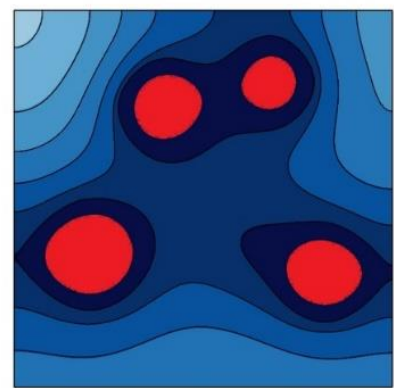

$\mathrm{T}_{2}$

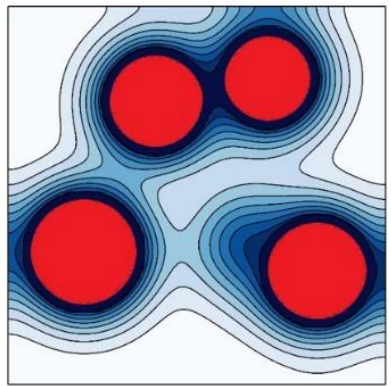

$\mathrm{T}_{1}$

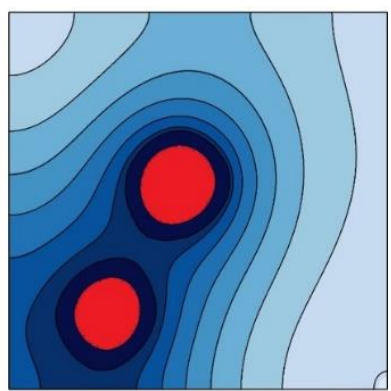

$\mathrm{T}_{2}-2$

(b)

Fig. 14: (a) Kinetic evolution of the volume fraction of $\beta$-phase and microstructure evolution; (b) characteristics of the concentration fields of B-component relative to the $\alpha$-phase during the dissolution process involving 7 particles of spherical shape and identical initial radius $r\left(t_{0}\right) / L=0.15$. The concentration fields of B-component $\bar{V}_{m} c_{B}^{\alpha}$ are drawn in the plane $z / L=0.32$ shown in $\mathrm{T}_{0}$ for different times $t . D / L^{2}: 0.004$ in $\mathrm{T}_{1}, 0.05$ in $\mathrm{T}_{2}$; and in the plane $z / L=0.75$ for $t . D / L^{2}=0.05$ in $\mathrm{T}_{3}$; simulation was performed using a mesh size of $100^{3}$ and time increment $\Delta t=10^{-6} \mathrm{~s}$. 


\section{Conclusion}

The Darken methodology, based on the intrinsic diffusion of components and on the drift velocity to which all compounds are subjected, has been shown in this work to be relevant to deal with diffusion-controlled phase transformations into binary two-phase systems. With the aim of obtaining a unique differential equation for component diffusion, we have developed this method $i$ ) in the framework of an algebraic VOF method allowing a sufficiently smooth interface function for spatial derivative expression through the interface, and ii) with the modeling strategy to express the intrinsic component fluxes thanks to the derivative of a diffusion potential which presents the advantage of being continuous through the interface. Moreover, this diffusion potential, which depends on the component concentrations and contains the equilibrium concentration information, is used to obtain an implicit resolution of the local interfacial equilibrium without any additional mass balance equation. Indeed, the verification results of this model on the $1 \mathrm{D}$ dissolution/precipitation problems show a very precise match between the concentration profiles and exact ones through the overall system, including the concentration jump at the interface.

From a kinetics point of view, the Darken drift velocity is shown to be the one that should advect the interface. The verification results have shown, here again, a precise match between simulated and analytical kinetics on the 1D and 3D geometries when the diffusion edge of the dissolved/precipitated particles are far from the boundaries. A first-order convergence for the interface displacement is obtained for the 1D case. In the case of the precipitation of a small spherical particle, the interaction of the diffusion edge with the domain boundaries induced a particle morphology change towards a cuboid-like shape. The same bifurcation from spherical particle shape is also simulated for the $3 \mathrm{D}$ precipitation and dissolution of 7 particles randomly distributed into the domain when the diffusion edges of each particle interacted with each other. The final particle shape can thus be related to the spatial distribution of the particles.

As for perspectives, the proposed Darken-based methodology for diffusion-controlled phase transformations can be easily extended to couple driving forces other than chemical ones, such as pressure or electric field, by introducing them into the diffusion potential. The coupling of these driving forces of mass transport is of interest, especially the mechano-chemical coupling involved in multiple material processing, which will require the resolution of the 
momentum conservation equation. Finally, High Performance Computing will also make it possible to consider a large number of particles in order to get closer to real systems for a better understanding of experimental processes.

Acknowledgments

We would like to thank the regional computing center MCIA (Mésocentre de Calcul Intensif Aquitaine) for the HPC resources.

\section{Bibliographie}

[1] D.A. Porter, Phase transformations in metals and alloys, 3rd ed., CRC Press, Boca Raton, Fla, 2009.

[2] Q. Lai, O. Bouaziz, M. Gouné, L. Brassart, M. Verdier, G. Parry, A. Perlade, Y. Bréchet, T. Pardoen, Damage and fracture of dual-phase steels: Influence of martensite volume fraction, Materials Science and Engineering: A. 646 (2015) 322-331. https://doi.org/10.1016/j.msea.2015.08.073.

[3] J.F. Li, P.A. Agyakwa, C.M. Johnson, Interfacial reaction in $\mathrm{Cu} / \mathrm{Sn} / \mathrm{Cu}$ system during the transient liquid phase soldering process, Acta Materialia. 59 (2011) 1198-1211. https://doi.org/10.1016/j.actamat.2010.10.053.

[4] S. Bordère, E. Feuillet, J.-L. Diot, R. de Langlade, J.-F. Silvain, Understanding of Void Formation in $\mathrm{Cu} / \mathrm{Sn}-\mathrm{Sn} / \mathrm{Cu}$ System During Transient Liquid Phase Bonding Process Through Diffusion Modeling, Metall and Materi Trans B. 49 (2018) 3343-3356. https://doi.org/10.1007/s11663-018-1391-8.

[5] D. den Ouden, L. Zhao, C. Vuik, J. Sietsma, F.J. Vermolen, Modelling precipitate nucleation and growth with multiple precipitate species under isothermal conditions: Formulation and analysis, Computational Materials Science. 79 (2013) 933-943. https://doi.org/10.1016/j.commatsci.2013.07.047.

[6] J. Herrnring, B. Sundman, B. Klusemann, Diffusion-driven microstructure evolution in OpenCalphad, Computational Materials Science. 175 (2020) 109236. https://doi.org/10.1016/j.commatsci.2019.109236.

[7] I. Steinbach, Phase-field models in materials science, Modelling Simul. Mater. Sci. Eng. 17 (2009) 073001. https://doi.org/10.1088/0965-0393/17/7/073001.

[8] S.G. Kim, W.T. Kim, T. Suzuki, Phase-field model for binary alloys, Phys Rev E. 60 (1999) 7186-7197. https://doi.org/10.1103/physreve.60.7186.

[9] T.L. van Noorden, C. Eck, Phase field approximation of a kinetic moving-boundary problem modelling dissolution and precipitation, Interfaces Free Bound. (2011) 29-55. https://doi.org/10.4171/IFB/247.

[10] B. Böttger, J. Eiken, M. Apel, Multi-ternary extrapolation scheme for efficient coupling of thermodynamic data to a multi-phase-field model, Computational Materials Science. 108 (2015) 283-292. https://doi.org/10.1016/j.commatsci.2015.03.003.

[11] C. Yang, S. Li, X. Wang, J. Wang, H. Huang, Phase-field simulation of multi-phase interactions in Fe-C peritectic solidification, Computational Materials Science. 171 (2020) 109220. https://doi.org/10.1016/j.commatsci.2019.109220. 
[12] S.K. Pabi, Computer simulation of the two-phase diffusion-controlled dissolution in the planar finite multilayer couples, Physica Status Solidi (a). 51 (1979) 281-289.

https://doi.org/10.1002/pssa.2210510133.

[13] T.C. Illingworth, I.O. Golosnoy, Numerical solutions of diffusion-controlled moving boundary problems which conserve solute, Journal of Computational Physics. 209 (2005) 207-225. https://doi.org/10.1016/j.jcp.2005.02.031.

[14] O. Olaye, O.A. Ojo, Leapfrog/Dufort-Frankel explicit scheme for diffusion-controlled moving interphase boundary problems with variable diffusion coefficient and solute conservation, Modelling Simul. Mater. Sci. Eng. 28 (2020) 015008. https://doi.org/10.1088/1361$651 \mathrm{X} / \mathrm{ab} 58 \mathrm{f} 3$.

[15] M. Falcone, D. Bothe, H. Marschall, 3D direct numerical simulations of reactive mass transfer from deformable single bubbles: An analysis of mass transfer coefficients and reaction selectivities, Chemical Engineering Science. 177 (2018) 523-536. https://doi.org/10.1016/j.ces.2017.11.024.

[16] D. Darmana, N.G. Deen, J.A.M. Kuipers, Detailed 3D Modeling of Mass Transfer Processes in Two-Phase Flows with Dynamic Interfaces, Chem. Eng. Technol. 29 (2006) 1027-1033. https://doi.org/10.1002/ceat.200600156.

[17] N. Balcázar-Arciniega, O. Antepara, J. Rigola, A. Oliva, A level-set model for mass transfer in bubbly flows, International Journal of Heat and Mass Transfer. 138 (2019) 335-356. https://doi.org/10.1016/j.ijheatmasstransfer.2019.04.008.

[18] D. Bothe, S. Fleckenstein, A Volume-of-Fluid-based method for mass transfer processes at fluid particles, Chemical Engineering Science. 101 (2013) 283-302. https://doi.org/10.1016/j.ces.2013.05.029.

[19] D. Bothe, M. Koebe, K. Wielage, J. Prüss, H.-J. Warnecke, Direct numerical simulation of mass transfer between rising gas bubbles and water, in: M. Sommerfeld (Ed.), Bubbly Flows, Springer Berlin Heidelberg, Berlin, Heidelberg, 2004: pp. 159-174. https://doi.org/10.1007/9783-642-18540-3_13.

[20] M.M. Francois, N.N. Carlson, The Balanced-Force Volume Tracking Algorithm and Global Embedded Interface Formulation for Droplet Dynamics With Mass Transfer, in: ASME 2010 3rd Joint US-European Fluids Engineering Summer Meeting: Volume 1, Symposia - Parts A, B, and C, ASMEDC, Montreal, Quebec, Canada, 2010: pp. 81-88. https://doi.org/10.1115/FEDSM-ICNMM2010-31032.

[21] H. Marschall, K. Hinterberger, C. Schüler, F. Habla, O. Hinrichsen, Numerical simulation of species transfer across fluid interfaces in free-surface flows using OpenFOAM, Chemical Engineering Science. 78 (2012) 111-127. https://doi.org/10.1016/j.ces.2012.02.034.

[22] D. Deising, H. Marschall, D. Bothe, A unified single-field model framework for Volume-OfFluid simulations of interfacial species transfer applied to bubbly flows, Chemical Engineering Science. 139 (2016) 173-195. https://doi.org/10.1016/j.ces.2015.06.021.

[23] D. Deising, D. Bothe, H. Marschall, Direct numerical simulation of mass transfer in bubbly flows, Computers \& Fluids. 172 (2018) 524-537. https://doi.org/10.1016/j.compfluid.2018.03.041.

[24] J.F. Li, P.A. Agyakwa, C.M. Johnson, A fixed-grid numerical modelling of transient liquid phase bonding and other diffusion-controlled phase changes, J Mater Sci. 45 (2010) 2340-2350. https://doi.org/10.1007/s10853-009-4199-8.

[25] L. Darken, Diffusion, Mobility and Their Interrelation through Free Energy in Binary Metallic Systems, Trans. AIME. 175 (1948) 184-201.

[26] S. Sridhar, A Commentary on "Diffusion, Mobility and Their Interrelation through Free Energy in Binary Metallic Systems,” L.S. Darken: Trans. AIME, 1948, vol. 175, p. 184ff, Metall and Mat Trans A. 41 (2010) 543-562. https://doi.org/10.1007/s11661-010-0177-7.

[27] A.D. Smigelskas, E.O. Kirkendall, Zinc diffusion in alpha brass, Trans. AIME. 171 (1947) 130142.

[28] M. Danielewski, B. Wierzba, Thermodynamically consistent bi-velocity mass transport phenomenology, Acta Materialia. 58 (2010) 6717-6727. https://doi.org/10.1016/j.actamat.2010.08.037. 
[29] J. Ågren, Diffusion in phases with several components and sublattices, Journal of Physics and Chemistry of Solids. 43 (1982) 421-430. https://doi.org/10.1016/0022-3697(82)90152-4.

[30] J. Andersson, J. Ågren, Models for numerical treatment of multicomponent diffusion in simple phases, Journal of Applied Physics. 72 (1992) 1350-1355. https://doi.org/10.1063/1.351745.

[31] B. Wierzba, Segregation in multicomponent mixtures under gravity: The bi-velocity method, Physica A. 390 (2011) 2325-2332. https://doi.org/10.1016/j.physa.2011.02.047.

[32] B. Wierzba, W.J. Nowak, Phase separation during the sedimentation process in $\mathrm{Cu}-\mathrm{Sn}$ alloy, Physica A: Statistical Mechanics and Its Applications. 523 (2019) 602-610. https://doi.org/10.1016/j.physa.2019.02.041.

[33] H. Larsson, A model for 1D multiphase moving phase boundary simulations under local equilibrium conditions, Calphad. 47 (2014) 1-8. https://doi.org/10.1016/j.calphad.2014.06.001.

[34] M.E. Glicksman, Diffusion in Solids: Field Theory, Solid-State Principles, and Applications, Wiley, 1999.

[35] H. Brenner, Kinematics of volume transport, Physica A: Statistical Mechanics and Its Applications. 349 (2005) 11-59. https://doi.org/10.1016/j.physa.2004.10.033.

[36] K.G.F. Janssens, D. Raabe, E. Kozeschnik, M.A. Miodownik, B. Nestler, Computational Materials Engineering: An Introduction to Microstructure Evolution, Elsevier Science, 2010.

[37] S. Bordère, J.-P. Caltagirone, A unifying model for fluid flow and elastic solid deformation: A novel approach for fluid-structure interaction, Journal of Fluids and Structures. 51 (2014) 344353. https://doi.org/10.1016/j.jfluidstructs.2014.09.010.

[38] Notus CFD Code, (n.d.). https://notus-cfd.org.

[39] C. Hirsch, Numerical Computation of Internal and External Flows: Computational Methods for Inviscid and Viscous Flows, volume 2, Wiley-Blackwell, 1990.

[40] C. Zener, Theory of Growth of Spherical Precipitates from Solid Solution, Journal of Applied Physics. 20 (1949) 950-953. https://doi.org/10.1063/1.1698258.

[41] M.J. Whelan, On the Kinetics of Precipitate Dissolution, Metal Science Journal. 3 (1969) 95-97. https://doi.org/10.1179/msc.1969.3.1.95.

[42] X. Guo, J. Sietsma, Y. Yang, Z. Sun, M. Guo, Diffusion-limited dissolution of spherical particles: A critical evaluation and applications of approximate solutions, AIChE J. 63 (2017) 2926-2934. https://doi.org/10.1002/aic.15676.

[43] T. Illingworth Charles, Modelling Diffusion Controlled Phases Changes, University of Cambridge, 2005.

[44] L.C. Brown, Diffusion-controlled dissolution of planar, cylindrical, and spherical precipitates, Journal of Applied Physics. 47 (1976) 449-458. https://doi.org/10.1063/1.322669. 